\title{
Aperçu de quelques paramètres qualitatives dans la caractérisation morpho-biométrique des poules locales (Gallus gallus) ou 'Batéké' dans les périphéries Nord et Sud de Brazzaville en République du Congo.
}

\author{
Hery Jolivet SAYA NGOUONIMBA ${ }^{(1)}$, OPOYE-ITOUA ${ }^{(2)}$ MOPOUNDZA Paul (2) \\ AKOUANGO Parisse \\ ${ }^{1}$ Enseignant chercheur au Lycée d'enseignement professionnel agricole amilcar cabral, Km 17 Brazzaville (LEPAAC). \\ ${ }^{2}$ Enseignant chercheur à l'école nationale des sciences agronomiques et forestières (ENS AF). \\ Département environnement et océanographie, Institut national de recherche en sciences exacts et naturelles (I.R.S.E.N). Institut \\ national de recherche en sciences exacts et naturelles
}

Mots-clés : paramètres qualitatifs, caractérisation morpho-biométrique, poule locale ou Batéké, périphérie nord et sud de Brazzaville.

Keywords: qualitative parameters, morpho-biometrics characterization, local hen or Batéké, North and South periphery of Brazzaville.

Publication date 30/11/2019, http://www.m.elewa.org/JAPS

\section{RESUME}

Cette étude portant sur l'aperçu de quelques paramètres qualitatives dans la caractérisation morpho-biométrique des poules locales (Gallus gallus) ou Batéké dans les périphéries Nord et Sud de Brazzaville avait pour but d'apprécier quelques aspects qualitatifs indispensables dans la caractérisation de ces poules dans les deux périphéries a été réalisée dans les périphéries nord et sud de Brazzaville comprenant respectivement les arrondissements 9 Djiri et 8 Madibou, s'est déroulée dans neuf (9) quartiers au total. A L'issu de cette étude, 929 poulet ont été échantillonnés dont 583 femelles et 346 mâles. Des enquêtes menées ont révélées que 651 poulet $(70 \%)$ ont une crête simple contre $278(30 \%)$ une crête double ; la couleur dominante de la crête est rouge, 922 poulet $(99 \%)$ contre 9 poulet $(1 \%)$ de crête rose pâle. 222 poulet $(24 \%)$, ont un bec jaune, 209 (22\%), ont un bec blanc, 154(17\%), ont un bec noir, 140(15\%), ont un bec corne, 131(14\%), ont un bec marron et $73(8 \%)$ un bec crème. Les barbillons sont majoritairement de forme ovale 922 , soit $(99 \%)$ contre $7(1 \%)$ de forme ronde ; la couleur dominante est le rouge à $100 \%$. Les yeux sont orange clair, 350 poulet $(37 \%)$, orange foncé, $200(22 \%)$, noir, $150(16 \%)$, yeux bruns 129, (14\%), yeux brun foncé, $100(11 \%)$. 378 poulet $(41 \%)$, ont des tarses blancs, $340(37 \%)$ ont des tarses jaunes, $86(9 \%)$, ont des tarses gris, $78(8 \%)$, ont des tarses pigmenté jaune et 37 (4\%)pigmenté noir. La distribution de la couleur a révélé que les phénotypes les plus nombreux sont : blanc (65 poulet soit 7\%), fauve uni (61 poulet soit $7 \%$ ), blanc tacheté (60 poulet soit $6 \%$ ) ; blanc herminé (60 soit $6 \%$ ), fauve à queue noir (51 poulet soit $5 \%$ ). La coloration du plumage est très variable, avec une fréquence élevée des couleurs blanc, fauve uni, blanc tâcheté et blanc herminé. Cette étude fait partie d'une série de travaux en cours de traitement sur les autres aspects qualitatifs et quantitatifs chez cette poule locale. 


\section{SUMMARY}

This study was focusing on the overvien of some qualitative parameters in the morphobiometrics characterization of local hens (Gallus gallus) in the North and South outskirts of Brazzaville was intended to appreciate some qualitative aspects essential in characterization of these hens in both peripheries has been carried out in the North and South outskirts of Brazzaville, comprising the districts 9 Djiri and 8 Madibou, respectively, in nine (9) quarters in total. At the outcome of this study, 929 chicken were sampled including 583 females and 346 males. Surveys conducted revealed that 651 chicken $(70 \%)$ have a single Crest against $278(30 \%)$ a double Ridge; the dominant color of the crest is red, 922 chicken $(99 \%)$ against 9 chicken (1\%) of pale pink crest. Also 222 chicken (24\%), have a yellow beak, $209(22 \%)$, have a white beak, $154(17 \%)$, have a Black beak, $140(15 \%)$ have a horn beak, $131(14 \%)$ have a Brown beak and $73(8 \%)$ a creamy beak. The barbels are predominantly oval in 922 , or $(99 \%)$ versus $7(1 \%)$ round shape; the dominant color is red at $100 \%$. The eyes are light orange, in 350 chicken (37\%), dark orange, $200(22 \%)$, black, 150 (16\%), Brown eyes 129, $(14 \%)$, dark brown eyes, 100 (11\%). 378 chicken (41\%), have white tarsi, 340 (37\%) have yellow tarsi, $86(9 \%)$, have grey tarsi, $78(8 \%)$, have yellow pigmented tarsi and $37(4 \%)$ Pigmented black. Color distribution revealed that the most numerous phenotypes were: white (65 chicken or 7\%), United Fawn (61 chicken 7\%), white spotted (60 chicken or 6\%); white hermined (60 or $6 \%$ ) and black-tailed Fawn (51 chicken or 5\%). The coloration of the plumage is very variable, with a high frequency of white colors, plain Fawn, white stained and white hermined. This study is part of a series of work being processed on other qualitative and quantitative aspects in this local chicken.

\section{INTRODUCTION}

Les poules locales ont survécu au long processus de la sélection naturelle qui a façonné les populations avicoles hétérogènes actuelles; ces populations diffèrent sur le plan morphologique et les caractères adaptatifs. Au Congo, comme dans la plupart des pays en en développement, les poules locales sont souvent classées en fonction de leurs phénotypes ou de leurs localisations géographiques. Elles sont élevées dans des systèmes totalement divagants, exprimant ainsi un faible niveau de performances (Akouango et al., 2004). L'aviculture familiale occupe une importante place dans les stratégies de lutte contre la pauvreté. Malgré la faible productivité, la forte exposition aux maladies, l'environnement difficile, les volailles locales représentent une activité primordiale en zone rurale pour l'obtention d'un revenu à travers la vente de la poule à maturité (J.C.Fotsa et al., 2010). Elle constitue aussi, une source importante de protéine et joue un rôle important sur le plan religieux, social et culturel (Gueye., 1998). En dépit de la concurrence de l'élevage moderne, l'aviculture traditionnelle continue de permettre aux populations particulièrement aux femmes de gagner leur vie dans des zones inhospitalières (Missohou et al., 2002). Les races locales, aux indéniables qualités de rusticité, méritent une autre attention sur le plan scientifique. La promotion de leur élevage et l'amélioration graduelle de leurs performances zootechniques peuvent être facteurs à la fois de développement économique et de sauvegarde de la biodiversité (Akonango et al., 2004). Une demande de produits issus des races locales dont les consommateurs préfèrent le goût plus intense de la viande et des œufs existe. Certaines races d'entres elles sont résistantes parasites et maladies tandis que d'autres sont capables de se développer dans des climats difficiles. Chaque race nécessite une description de ses caractéristiques physiques, de ses caractères de production, de sa répartition géographique, de son utilisation principale, de l'effectif de sa population, de sa conservation ex 
situ et la connaissance indigène qui lui est spécifique. L'élevage de la poule locale demande très peu d'investissement et est une activité économique pour beaucoup de familles vivant dans les zones rurales et même depuis des décennies dans les zones urbaines et périurbaines. Il peut offrir incontestablement, par son cycle de production court, une solution rapide et abordable par le plus grand nombre, de plus une grande partie de la population pratique déjà ce type d'élevage de manière traditionnelle (N.Moula et al., 2011a). Le développement des filières avicoles (rustiques en particulier et améliorée) fait face à de nombreuses contraintes, telles que la disponibilité des intrants et infrastructures d'élevage, l'accès limité aux soins vétérinaires prophylactiques et curatifs, aux crédits et connaissances qui permettraient d'améliorer la productivité de cette race locale. L'élevage des poules locales ne peut, par contre, satisfaire la demande nationale, en œufs, en viandes des volailles industrielles. Notons que la poule Batéké n'a jamais fait l'objet d'une étude de variabilité génétique. Des stratégies de gestion et de valorisation des ressources avicoles locales

\section{METHODOLOGIE :}

La collecte des données s'est déroulée dans les neuf (9) quartiers que comptent les périphéries Nord et Sud dont dépendent les arrondissements 8 Madibou et 9 Djiri de la ville de Brazzaville.

L'enquête a été conduite chez les éleveurs et propriétaires des poules en deux phases :

- la première phase ou méthode dite passive, celle de la prospection qui consiste à localiser les élevages de poules locales et parcelles possédant ces dernières; par la suite un entretien superficiel sur les raisons de cette étude avec le propriétaire des poules débouchant sur un rendez-vous.

- la deuxième phase ou méthode dite active, celle après un recensement des parcelles abritant l'élevage des poules de races locales. Nous avons reparti les jours selon la disponibilité de chacun afin de réaliser notre travail. Nous avons ainsi élaboré une fiche sont donc nécessaires, à la fois, pour le développement économique rural et la sauvegarde de la biodiversité par la connaissance détaillée préalable de ces ressources et leurs performances (Fatima Zohra M. et al., 2014); ainsi in en ait de même pour la poule Batéké. La caractérisation phénotypique et génétique de cette population avicole traditionnelle n'a pas été faite de manière systématique dans les majorités des départements du Congo-Brazzaville, en dehors de l'étude des phénotypes et des performances d'élevage chez des populations locales de volailles du genre Gallus gallus au Congo et la Croissance pondérale et productivité de la poule locale Gallus domesticus en élevage fermier au Congo réalisée par Akonango et al. Cette étude a pour objectif d'approfondir la connaissance sur les analyses morphobiométriques et la diversité phénotypique et génétique des populations des poules locales dans les périphéries nord et sud de Brazzaville. A long terme, ces informations devraient contribuer à la revalorisation du sous-secteur avicole par l'appui aux éleveurs qui sont habitués à l'élevage de cette race.

d'enquête qui nous a permis de travailler. Au total, un effectif de 929 poules dont 346 mâles et 584 femelles ont été recensé et étudiées parmi les 46 éleveurs visités dans les quartiers de la périphérie sud et 34 dans les quartiers de la périphérie Nord de Brazzaville. Chaque poule choisie a été décrit phénotypiquement. Les caractéristiques visuelles ont porté sur: la couleur et la forme du bec; la couleur des yeux; le type, et la coloration de la crête; la coloration et la forme des barbillons; la couleur, répartition, structure et le motif du plumage; la couleur des tarses. En dehors de ces paramètres phares, il a été relevé les caractéristiques de l'éleveur de l'éleveur et de son élevage (nom du quartier, points GPS, numéro de la poule, sexe, origine, race) et les caractéristiques socio-économiques des éleveurs de poules de races locales. Toutes ces mesures sont prises en présence de l'éleveur ou du 
propriétaire qui fournit les informations nécessaires pour la bonne marche de cette étude. A la fin, chaque poule était photographiée afin de réaliser une description plus précise pour la couleur de son plumage, le type de crête et couleur des yeux, la coloration des tarses, la prise du poids corporel. Chaque sujet a fait l'objet d'une description détaillée directe portant sur les caractères qualitatifs portant sur la couleur et la forme du bec; la couleur des yeux; le type, et la coloration de la crête ; la coloration et la forme des barbillons ; la couleur, répartition, structure et le motif du plumage; la couleur des tarses selon la nomenclature de Coquerelle., (2000). Chaque poule était photographiée afin de réaliser la description précise du plumage. Une analyse descriptive des données qualitatives sur la caractérisation morpho-métrique et profil phénotypique des poules locales ou Batéké a été faite à l'aide du logiciel $<<\mathrm{R}>>$.

4 RESULTATS :

4.1 Type et coloration de la crête :

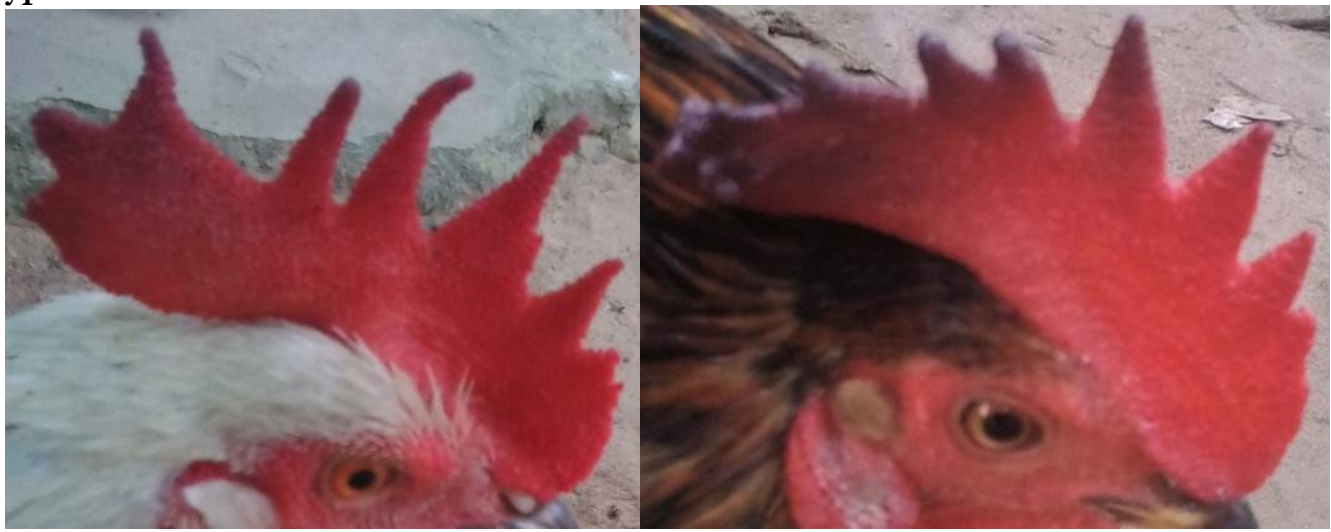

Figure n¹: Photographie de la crête simple

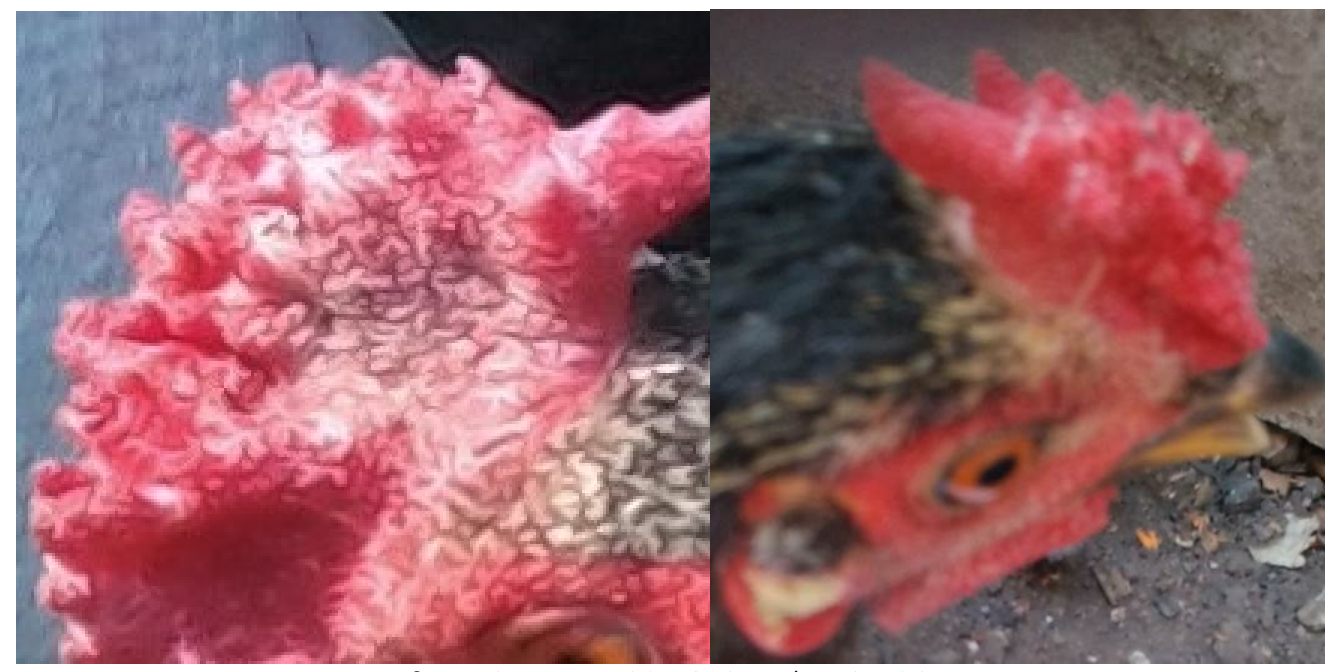

Figure $\mathbf{n}^{\circ} 2$ : Photographie de la crête double ou en V 


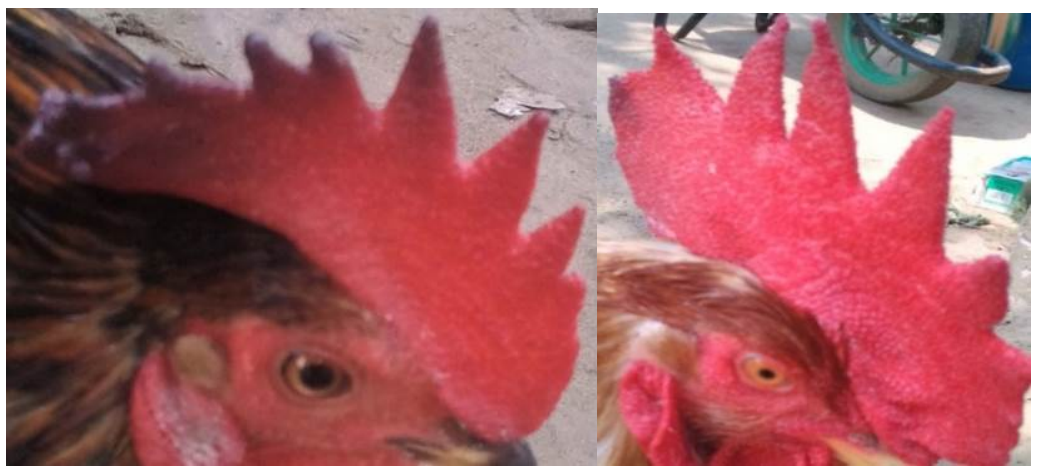

Figure $n^{\circ}$ 3: Photographie crête de couleur rouge

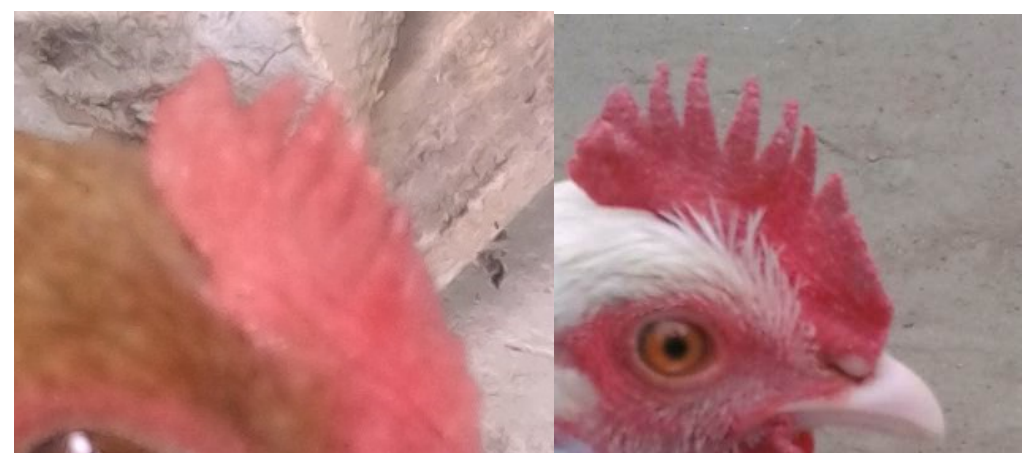

Figure n 4 : Photographie de la crête rose pâle

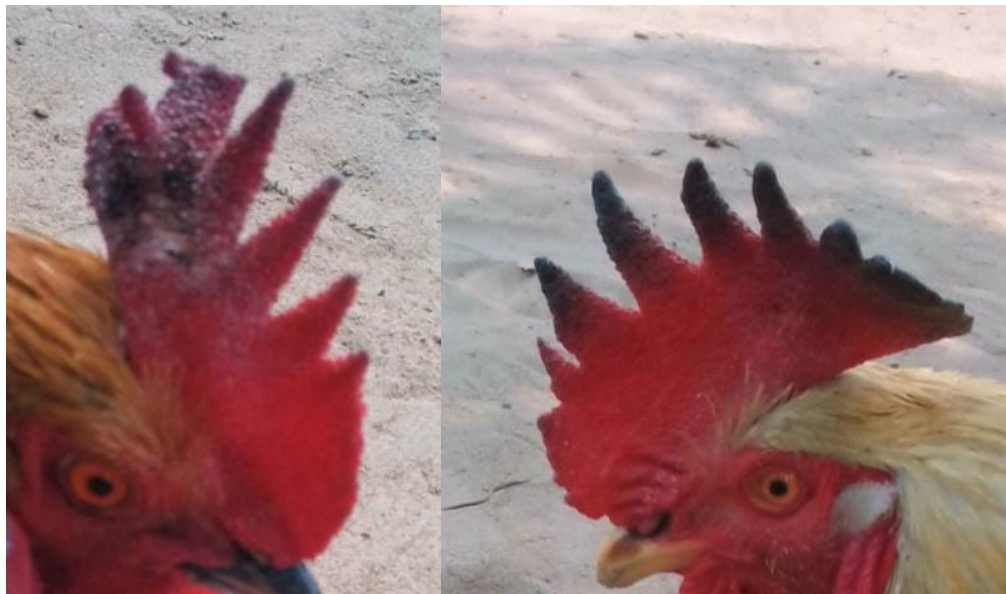

Figure $n^{\circ} \mathbf{5}$ : Photographie de la crête rouge avec pigmentation noir (ou crête rouge sablé)

L'observation des types de crêtes chez les poules dans les périphéries nord et sud de Brazzaville à révéler que sur 929 sujets observés, $651(70 \%)$ ont la crête simple et $278(29,92 \%)$ ont la crête double. Selon la distribution du plumage, pour la crête simple toutes les couleurs de plumages sont représentés avec comme dominantes le blanc, le blanc tacheté, le fauve uni, le type perdrix et le noir étendu ; tandis que pour la crête double, quelques plumages représentés tels que: le blanc, le type perdrix, le noir gris perle, le blanc herminé et le doré à liséré noir. Concernant la coloration de la crête, sur 929 sujets observés, $922(99 \%)$ ont une crête rouge et $9(1 \%)$ des sujets ont une crête rose pâle. 


\section{Variation du crête rouge selon la couleur du plumage}

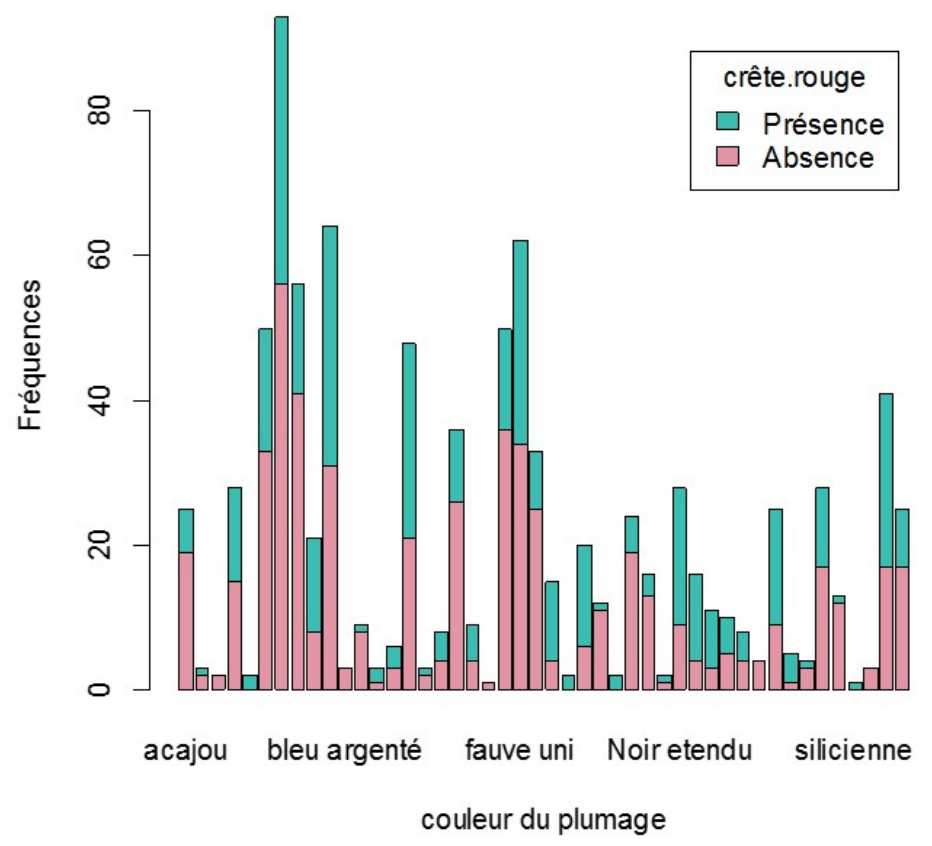

Variation du crête rouge pale selon la couleur du plumag

Figure $\mathbf{n}^{\circ} \mathbf{6}$ : variation de la crête rouge selon la couleur du

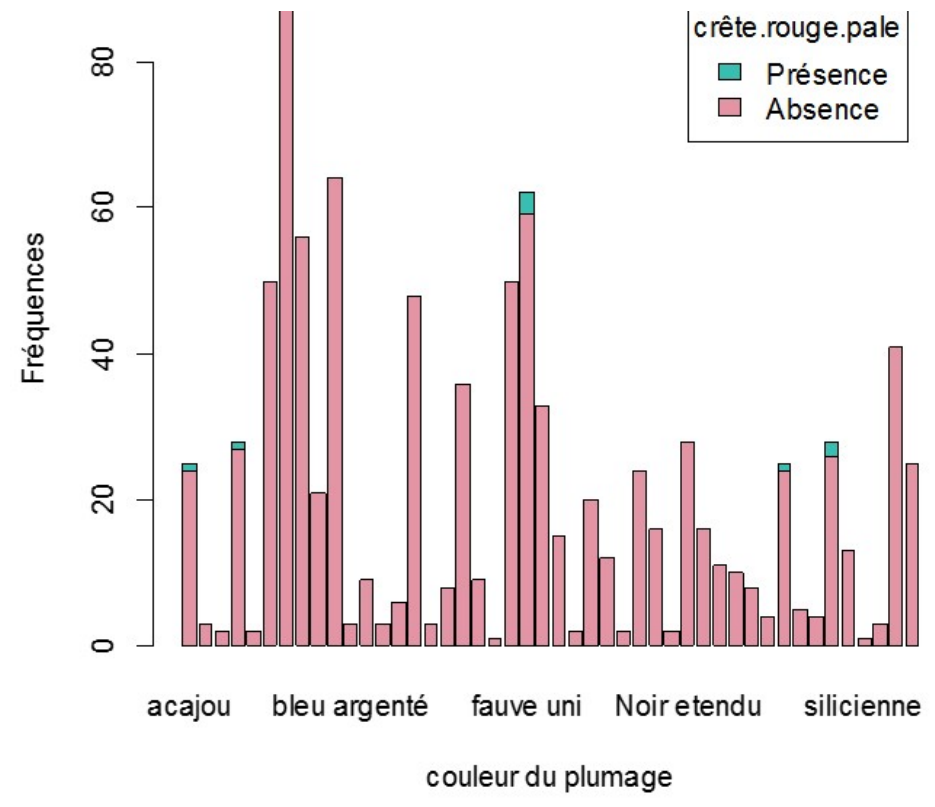

Figure $n^{\circ} 7$ : variation de la couleur rouge pâle 


\subsection{Coloration du bec :}

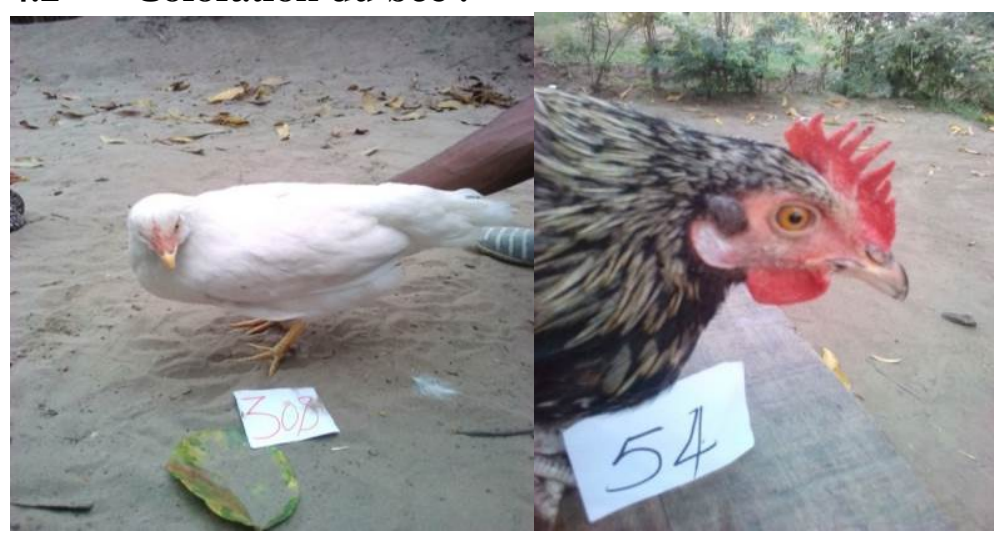

Figure $\mathbf{n}^{\circ} \mathbf{8}$ : Photos Bec blanc

figure n 9: Photos Bec Corne

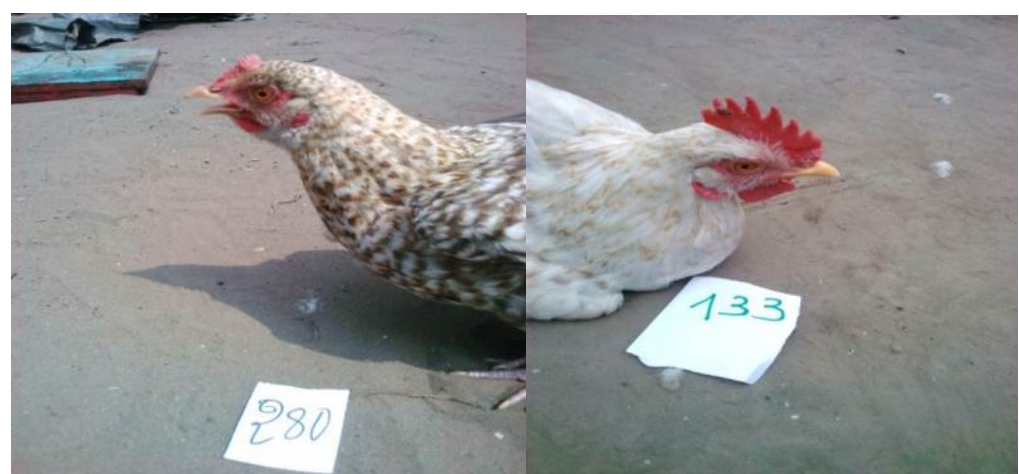

Figure n 10 : Photos Bec Crème $\quad$ figure $\mathbf{n}^{\circ} 11$ : Photos Bec jaune

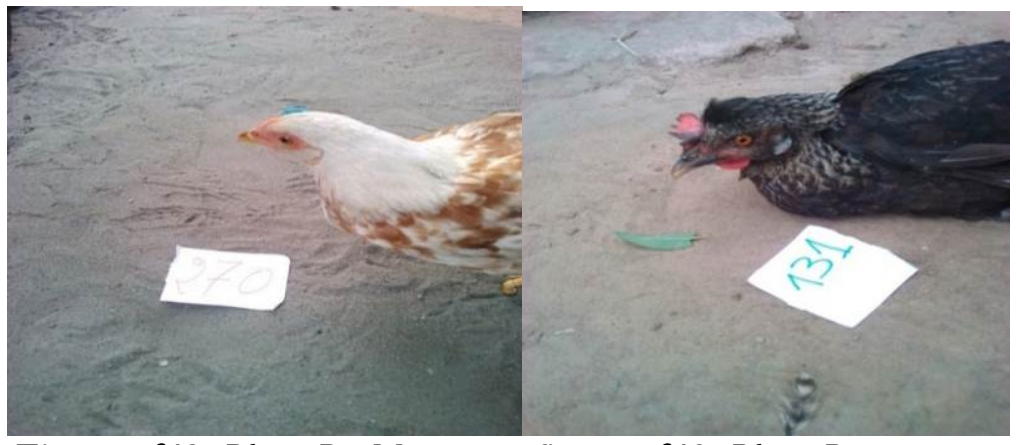

Figure n'012: Photos Bec Marron

figure n¹3: Photos Bec noir

L'observation des becs chez les poules dans les périphéries nord et sud de Brazzaville a révélé que sur 929 sujets observés, 222 soit $23,89 \%$ ont un bec jaune, 209 soit $22 \%$ ont un bec blanc, 154 soit $16,57 \%$ ont un bec noir , 140 soit $15 \%$ ont un bec corne , 131 soit $14 \%$ ont un bec marron et 73 soit $7,85 \%$ ont un bec crème. Dans la coloration du bec blanc, les plus représentatifs sont le fauve à queue noir, blanc, type perdrix, blanc herminé, acajou. Dans la coloration du bec corne, les plus représentatifs sont blanc tacheté, blanc, fauve à queue noir, noir à camail doré et fauve uni. Dans la coloration du bec crème, les plus représentatifs sont doré saumoné bleu, blanc, blanc sale, fauve à queue noir et type perdrix. Dans la coloration du bec jaune, les plus représentatifs sont blanc herminé, coucou, barré, blanc et fauve uni. 
4.3 Coloration et forme des barbillons :

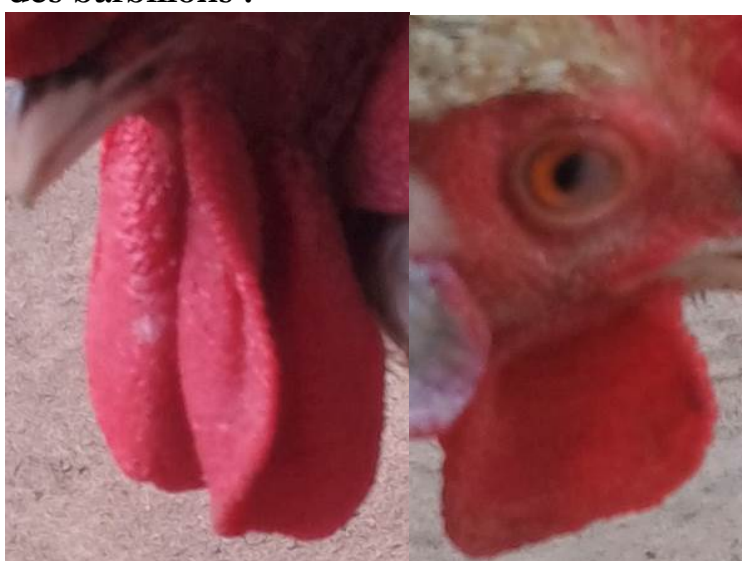

Figure $n^{\circ} 14$ : barbillons ovale

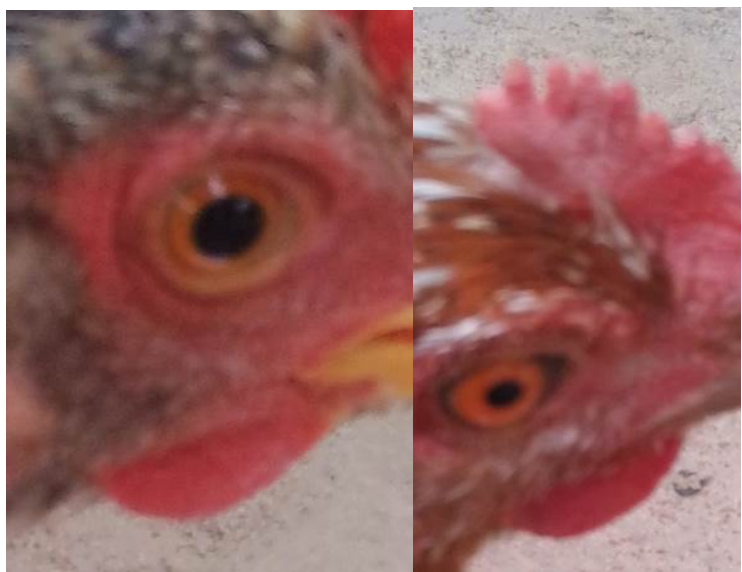

Figure $n^{\circ} 15$ : barbillons ronde

Pour un effectif de 929 sujets caractérisés, 922 ont un barbillon de forme ovale $(99 \%)$ contre 7 sujets soit $(1 \%)$ ont un barbillon de forme ronde. La couleur est rouge à 100\%. Les phénotypes dominants sont: blanc, fauve uni, blanc tacheté, blanc herminé et fauve à queue noir.

\subsection{Coloration des yeux :}

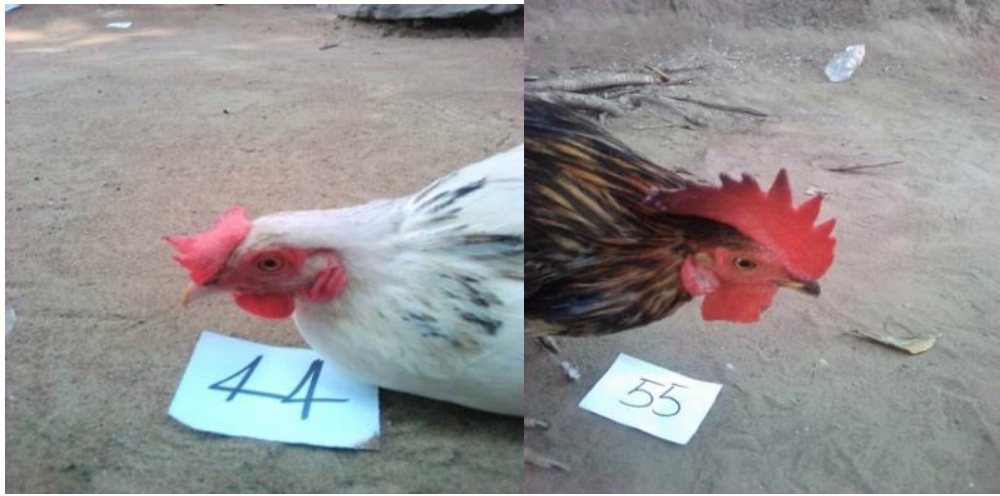

Figure $\mathbf{n}^{\circ} \mathbf{1 6}$ : Photos yeux Orange clair Figure n¹7 : Photos yeux Orange foncé 


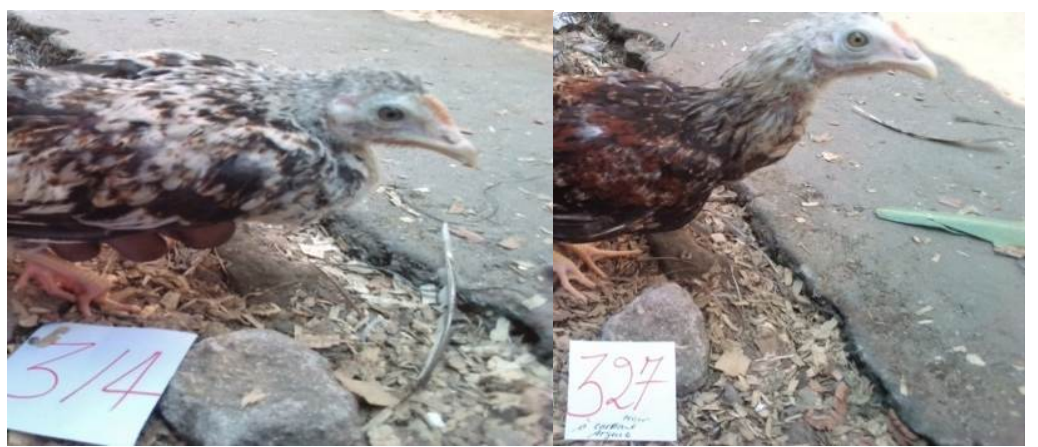

Figure $\mathbf{n}^{\circ} 18$ : Photos brun foncé Figure $\mathbf{n}^{\circ} 19$ : Photos brun clair

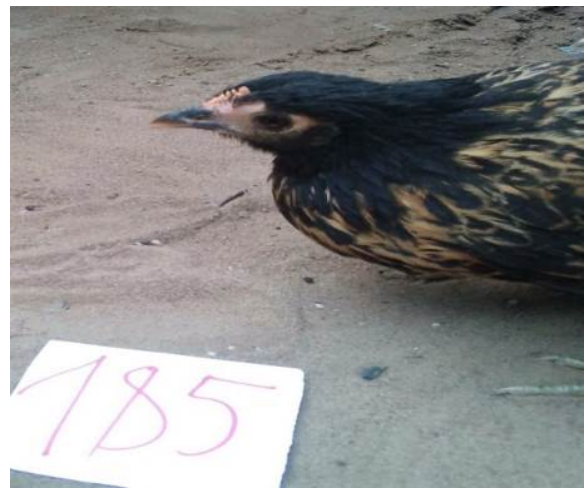

Figure $\mathrm{n}^{\circ} 20$ : Photos yeux Noir

Sur 929 sujets observés, 350 soit $(36,67 \%)$ ont des yeux orange clair ; 200 sujets soit $(21,52 \%)$ ont des yeux orange foncé; 150 sujets soit
$(16,14 \%)$ ont des yeux noirs ; 129 sujets soit $(13,88 \%)$ ont des yeux brun clair et 100 sujets soit $(10,76 \%)$ ont des yeux brun foncé.

\subsection{Coloration des tarses :}

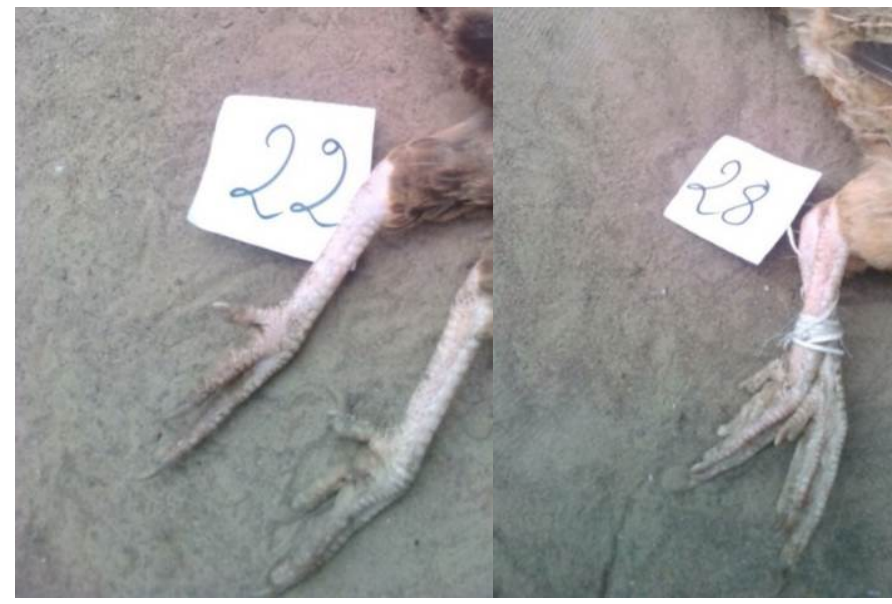

Figure n²1 : Photos tarse de couleur blanche 


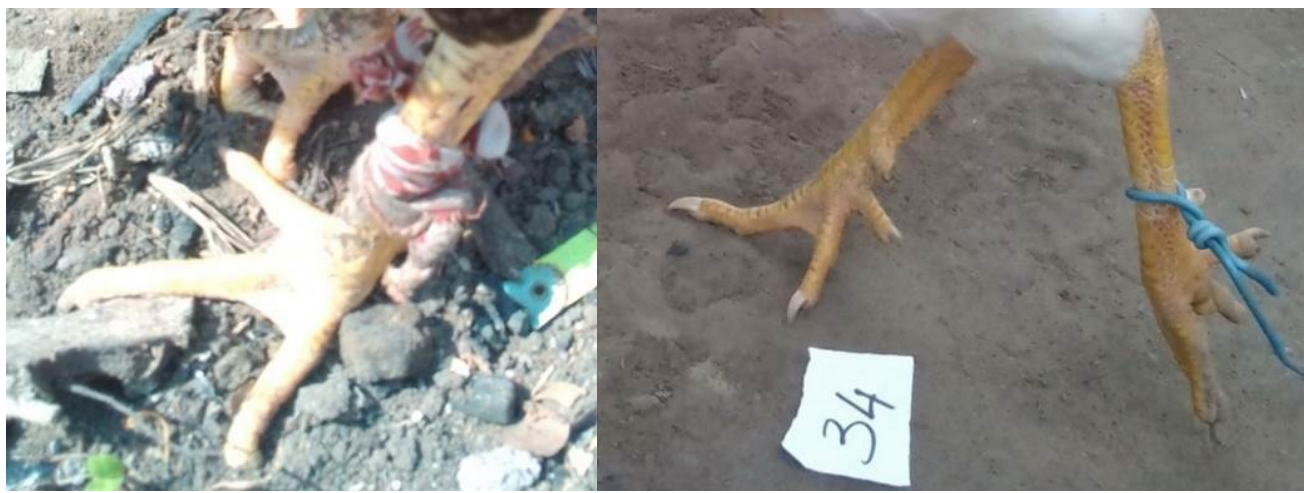

Figure $\mathbf{n}^{\circ} 22$ : Photos tarses de couleur jaune

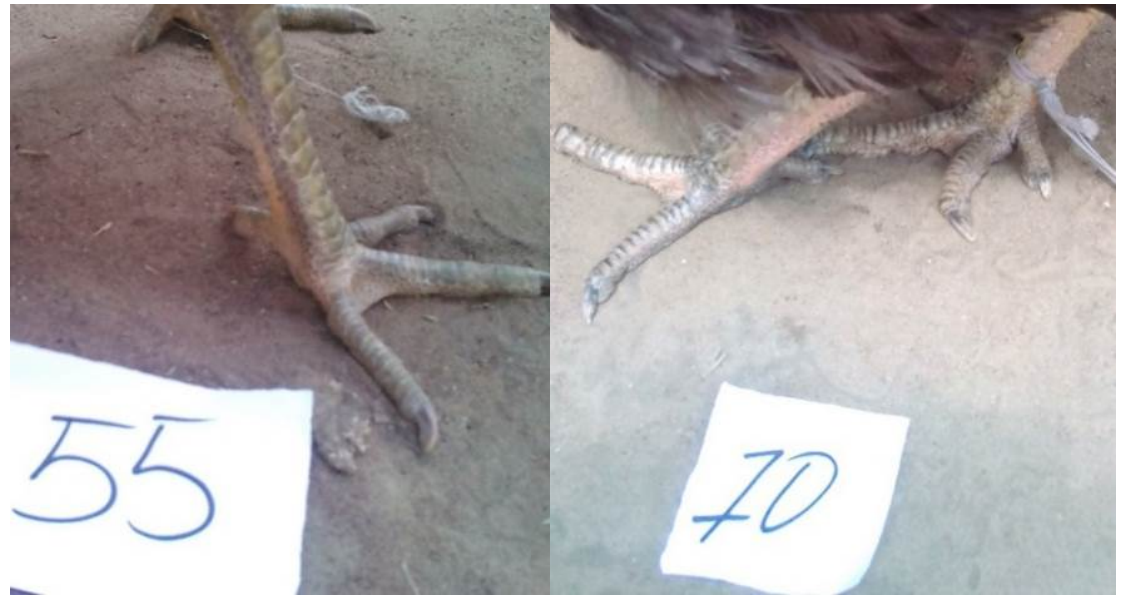

Figure $\mathbf{n}^{\circ} 23$ : Photos tarse pigmenté jaune

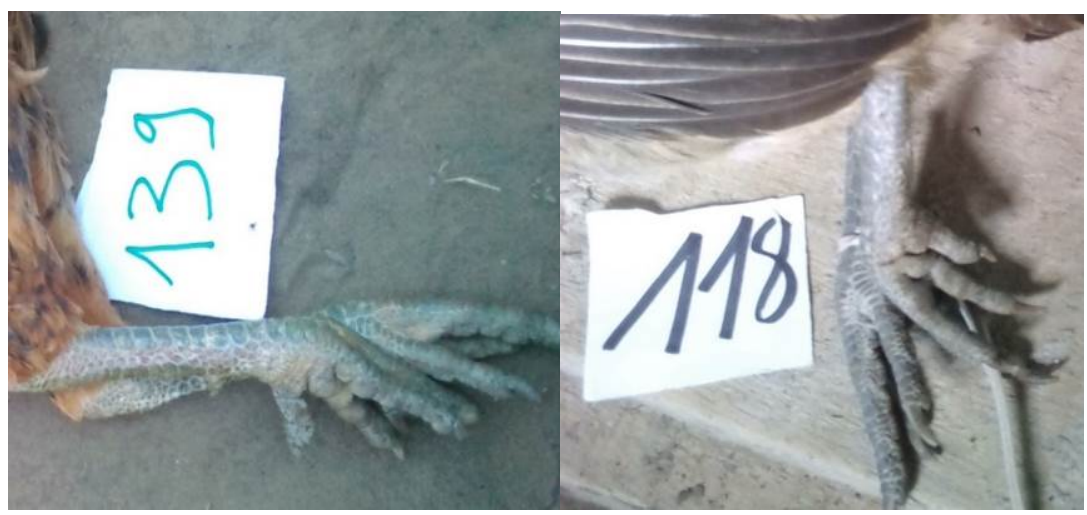

Figure $\mathbf{n}^{\circ} 24$ : Photos tarse pigmenté noir 


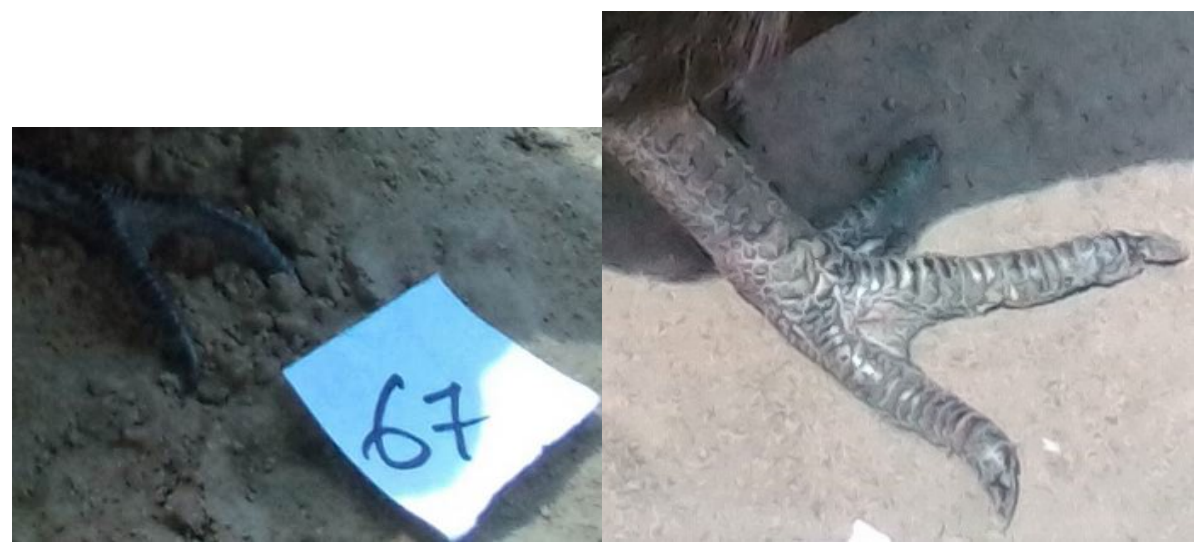

Figure $\mathbf{n}^{\circ} 25$ : Photos tarse fibro-mélanose ou nègre

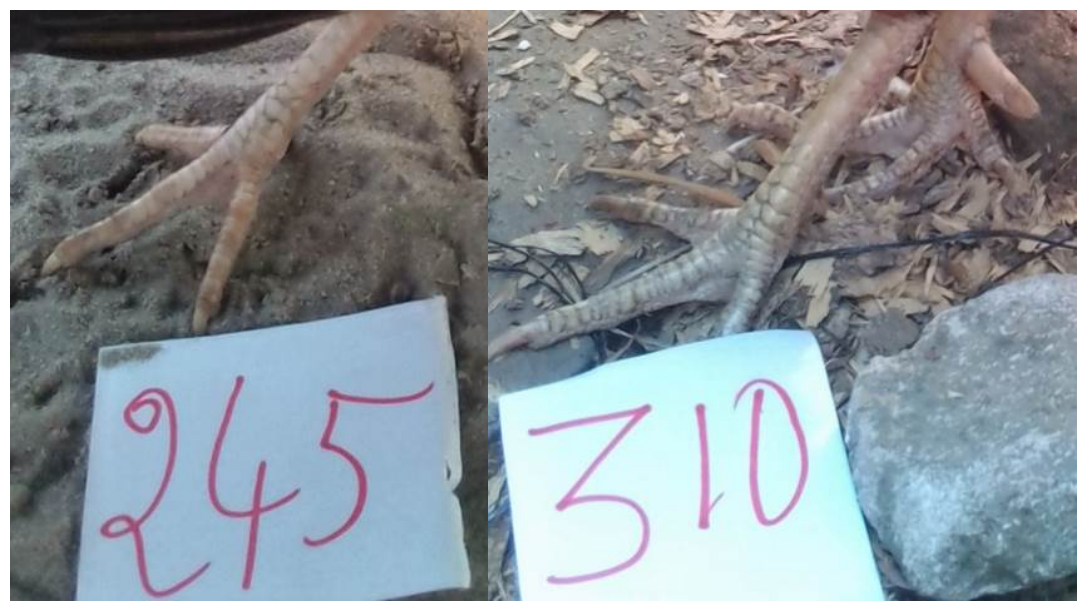

Figure $\mathbf{n}^{\circ} 26$ : Photos tarse rose pale 


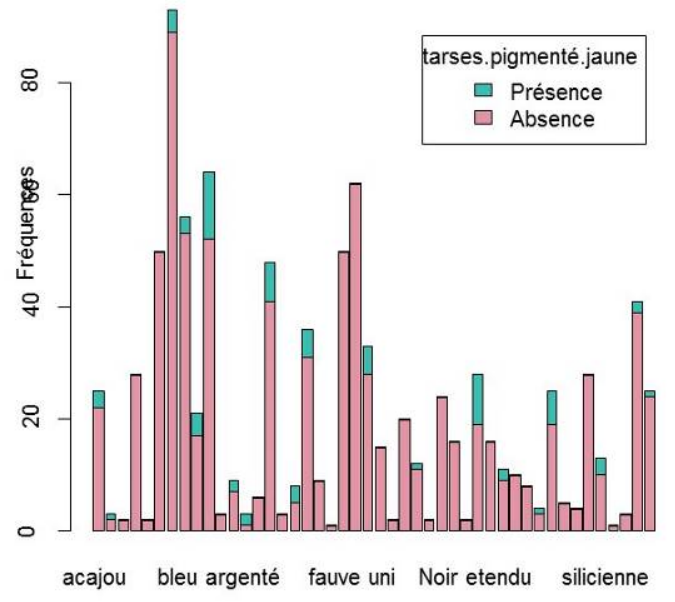

Figure $\mathbf{n}^{\circ} 27$ : tarses pigmenté jaune

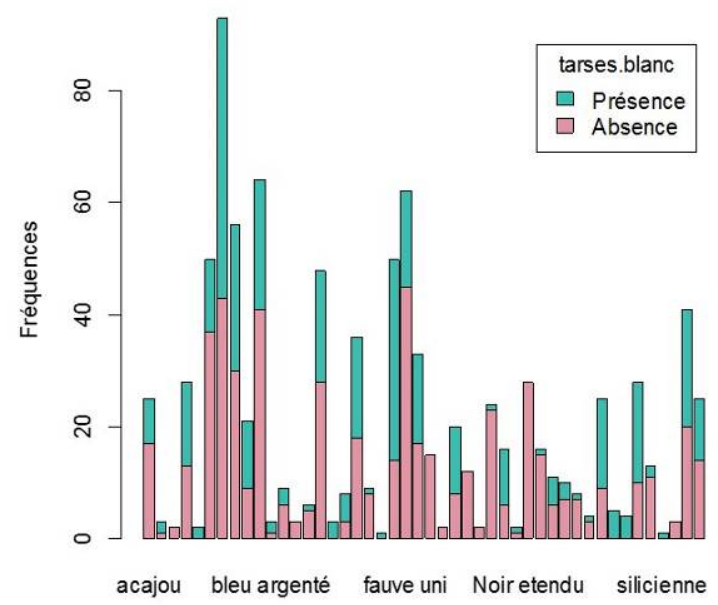

Figure $n^{\circ} 28$ : tarses blanc

Variation de tarse gris selon la couleur du plumage

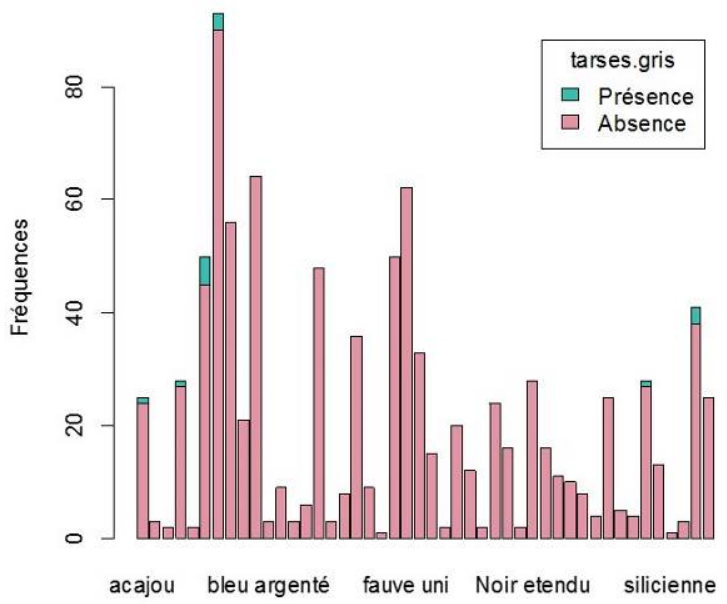

Figure $n^{\circ} 29$ : tarses gris
Variation de tarse jaune selon la couleur du plumage

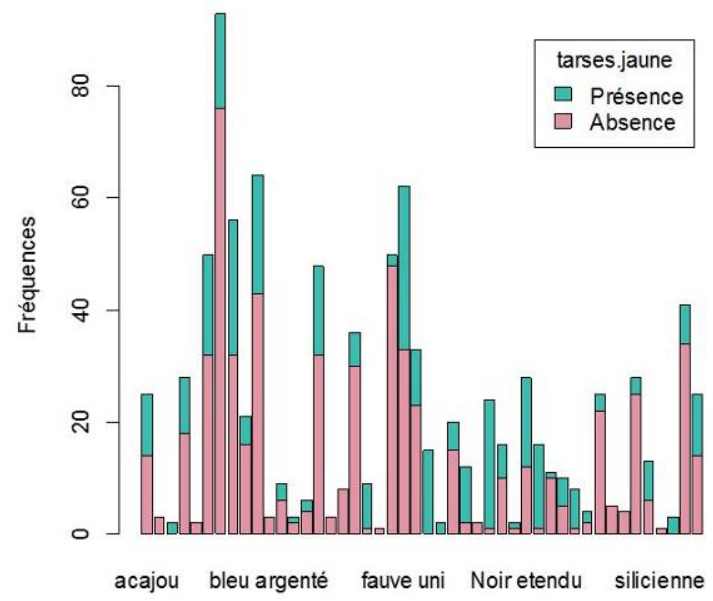

Figure $\mathrm{n}^{\circ} \mathbf{3 0}$ : tarses jaune 
Variation de tarse noir selon la couleur du plumage

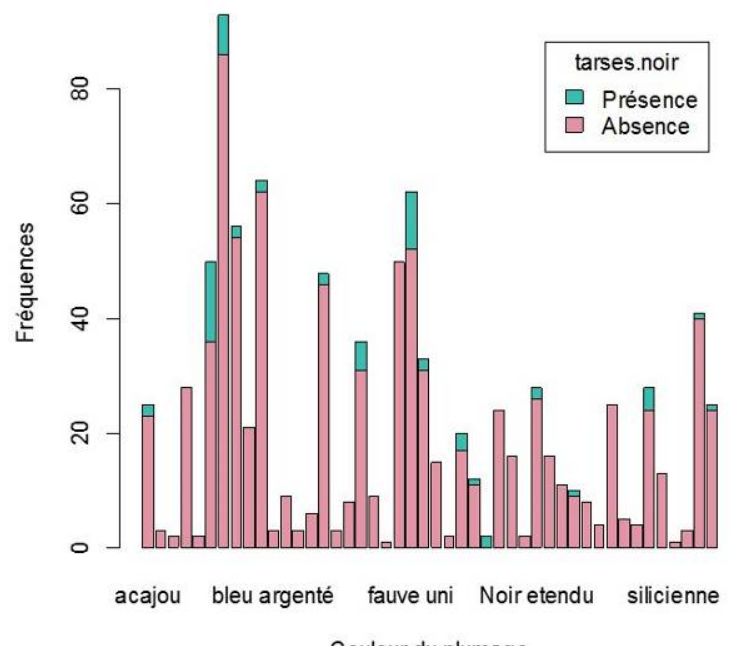

Figure $\mathbf{n}^{\circ} \mathbf{3 1}$ : tarses noir
Variation de tarse rose.pale selon la couleur du plumage

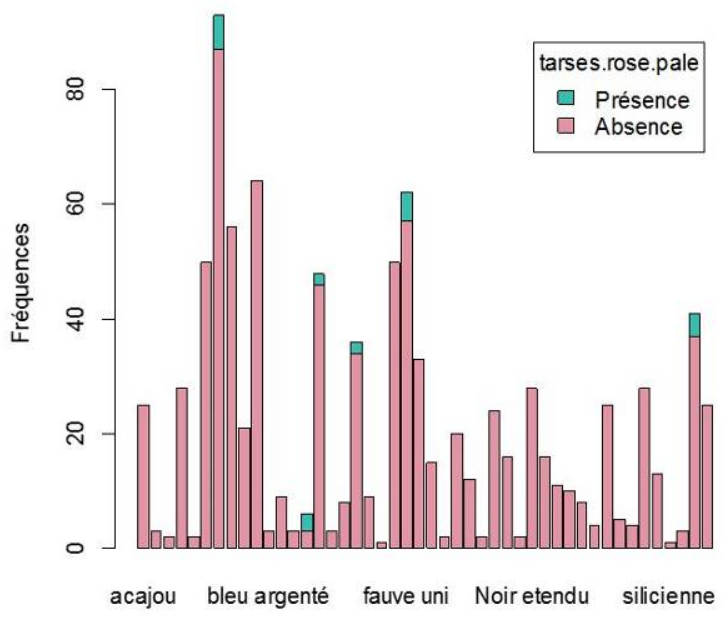

Figure $n^{\circ} 32$ : tarses rose- pâle

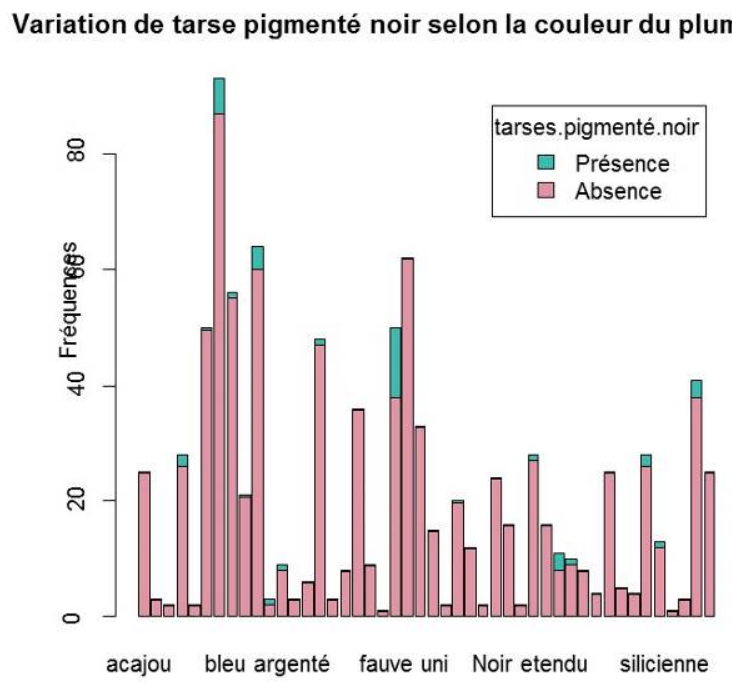

Figure $\mathbf{n}^{\circ} 33$ : tarses pigmenté noir

La caractérisation de la couleur des tarses par rapport à la couleur du plumage révèle que sur les 929 sujets, il ya 378 Blanc (40,68\%), 340 jaune $(36,59 \%), 86$ gris $(9,25 \%), 78$ pigmenté jaune $(8,39 \%)$ et 37 pigmenté noir $(3,98 \%)$ comme couleurs dominantes sur les 45 coloris rencontrées sur le terrain. Dans la coloration tarses blanche, les couleurs de plumage qui dominent sont le blanc avec, le fauve à queue noir, le blanc herminé, le blanc tacheté et enfin le type perdrix. Dans la coloration tarses pigmenté jaune, les plus représentatifs sont le blanc tacheté, le noir étendu, le coucou, le froment, et le blanc. Dans la coloration tarses gris, les plus représentatifs sont le blanc, le type perdrix, la wyandotte argenté, le saumoné doré, l'Acajou. Dans la coloration tarses jaune, les plus représentatifs sont fauve uni, blanc herminé, noir à camail doré, blanc tacheté, barré. Dans la coloration tarses noir, les plus représentatifs sont barré, fauve uni, blanc, doré saumoné bleu et enfin saumoné doré. Dans la 
coloration tarses rose pale, les plus représentatifs sont blanc, fauve uni, type perdrix, cou-nu et enfin coucou. Dans la coloration tarses pigmenté noir, les plus représentatifs sont fauve a queue noir, blanc, type perdrix, blanc tacheté, noir restreint.

5 Distribution de la couleur de plumage par rapport au phénotype :
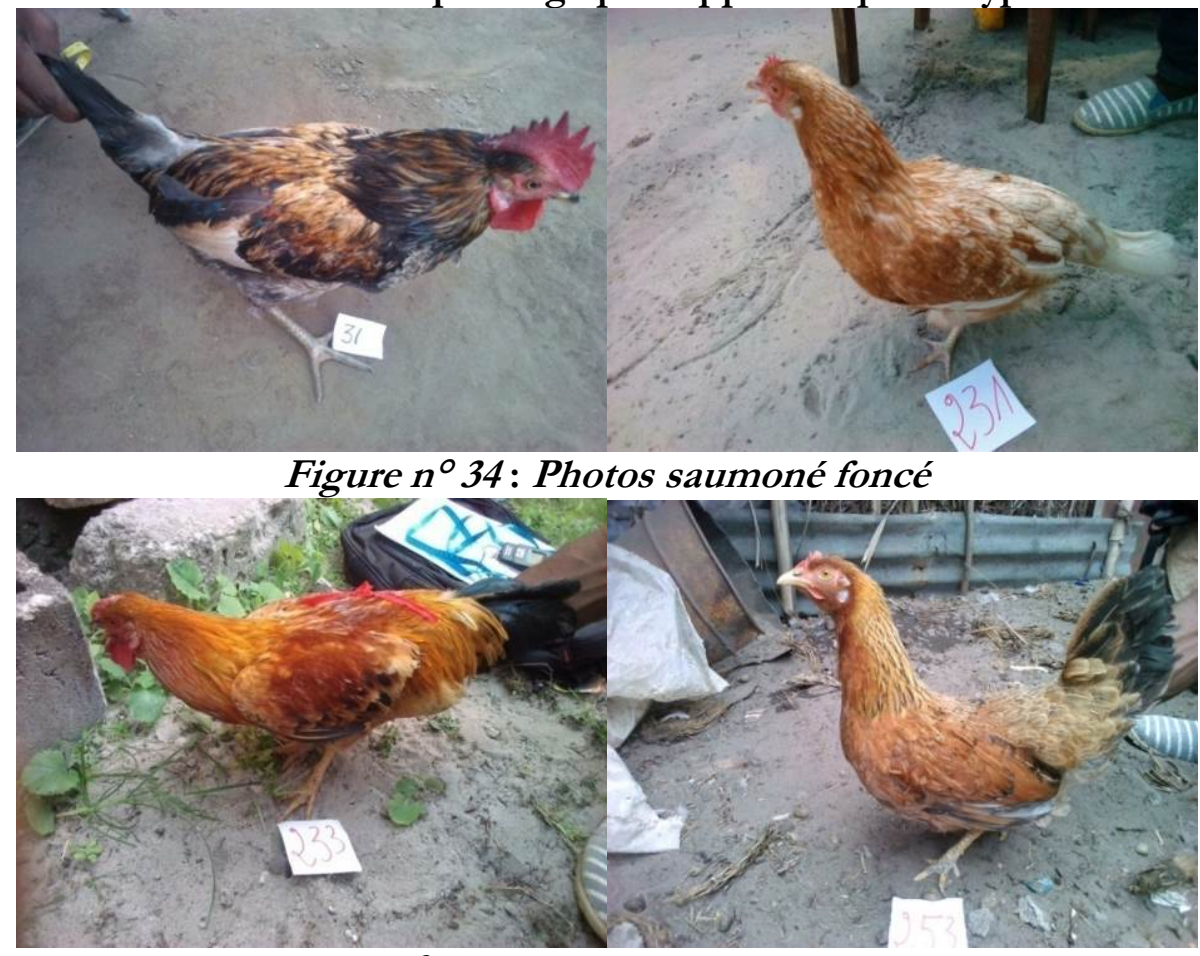

Figure n⿳35: Photos fauve à queue noir

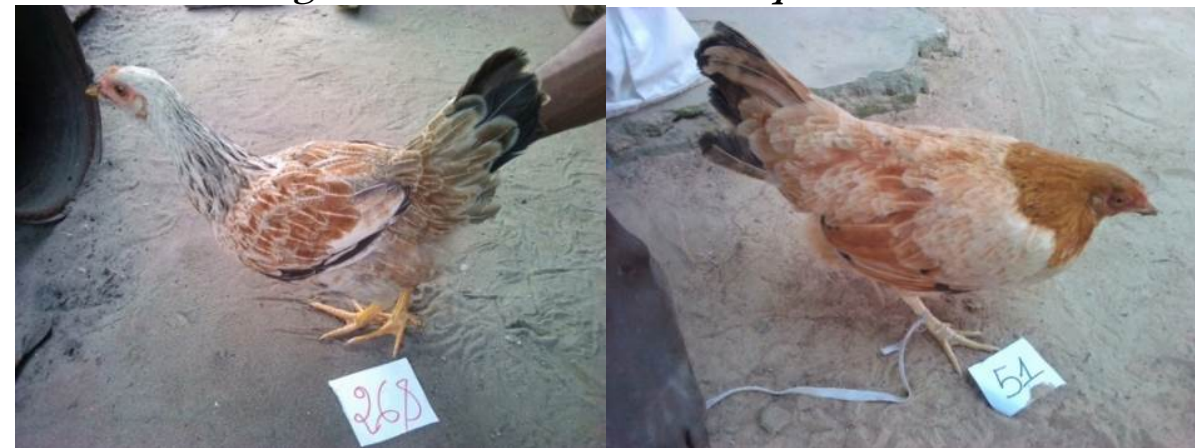

Figure no36: Photos froment

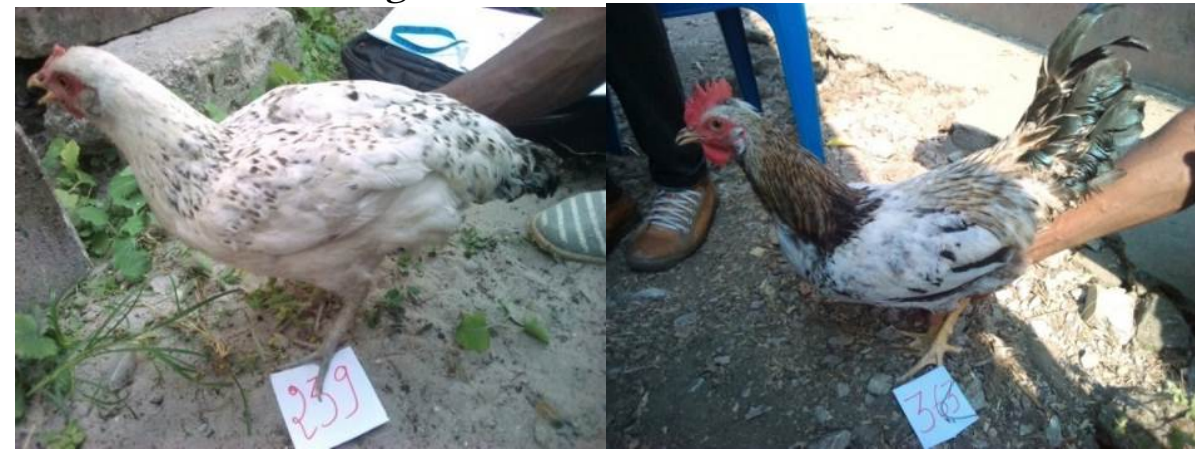

Figure n³7: Photos herminé 


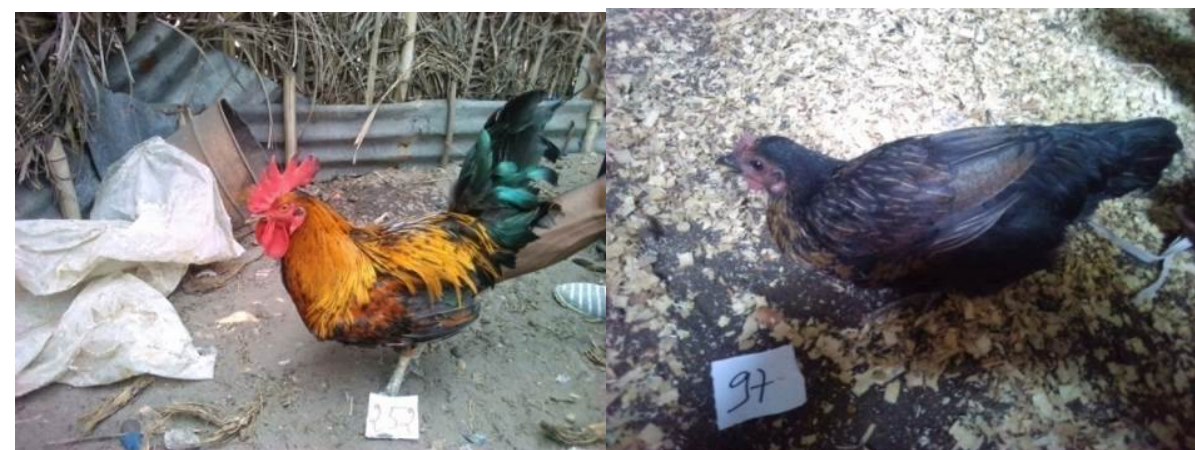

Figure n⿳38: Photos noir à camail doré

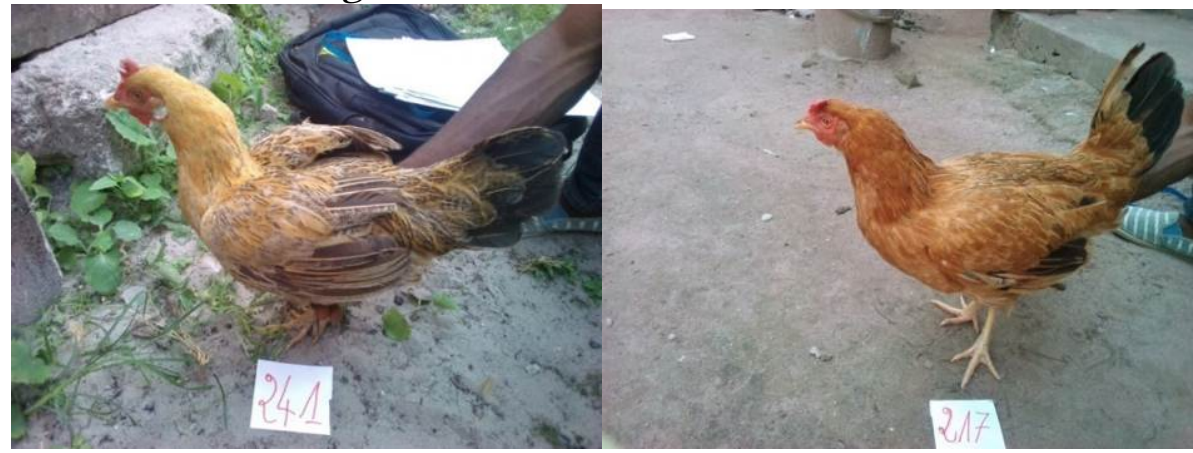

Figure nº39:Photoskokloyaya

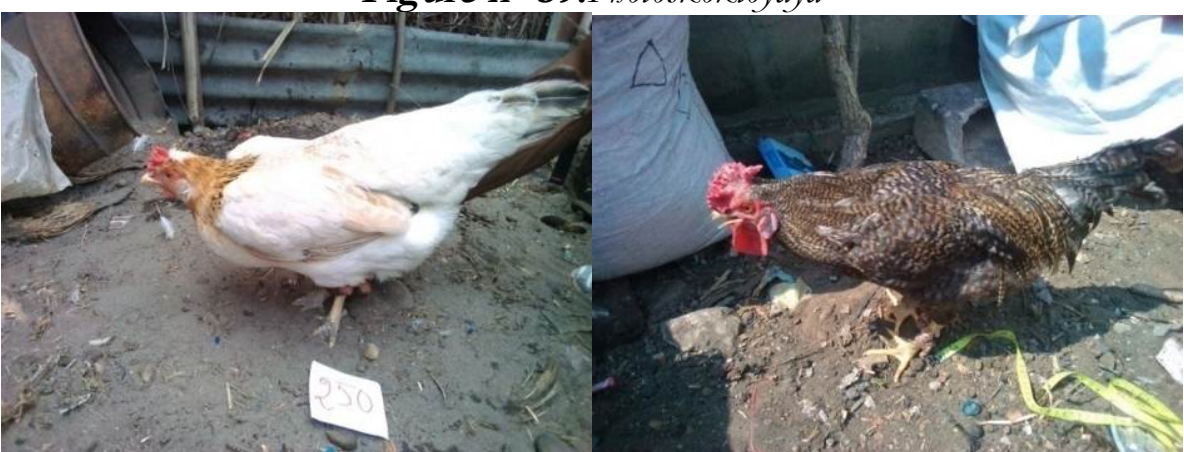

Figure $\mathbf{n}^{\circ} \mathbf{4 0}:$ Photos wyandotte argenté à liseré

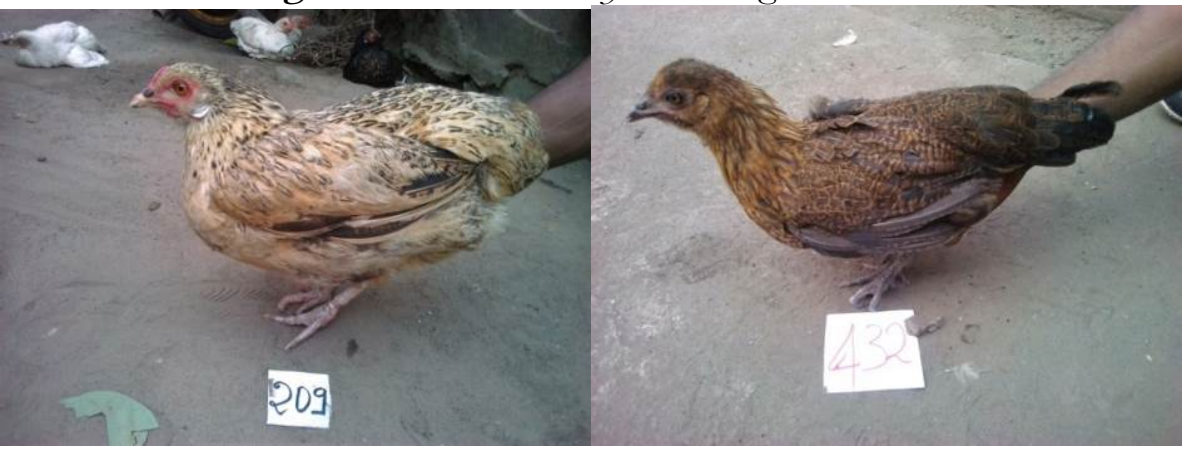

Figure n²1: Photos perdrix maillé doré 


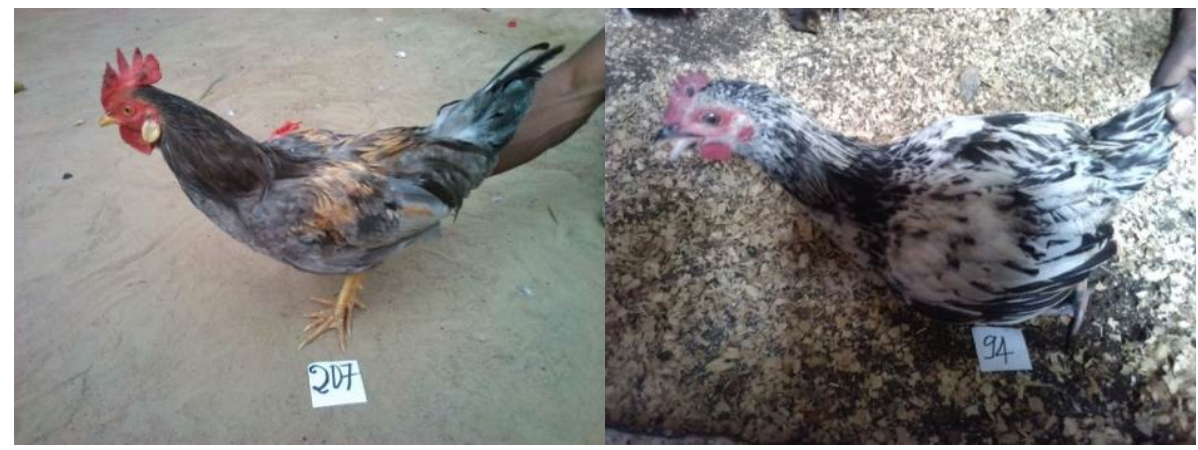

Figure n²: Photos doré saumoné bleu

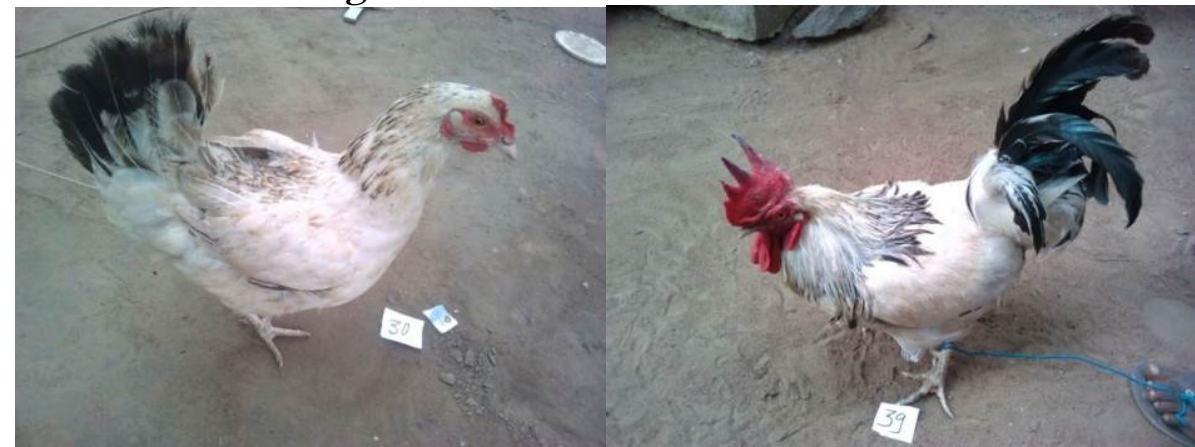

Figure n³: Photos blanc berminé

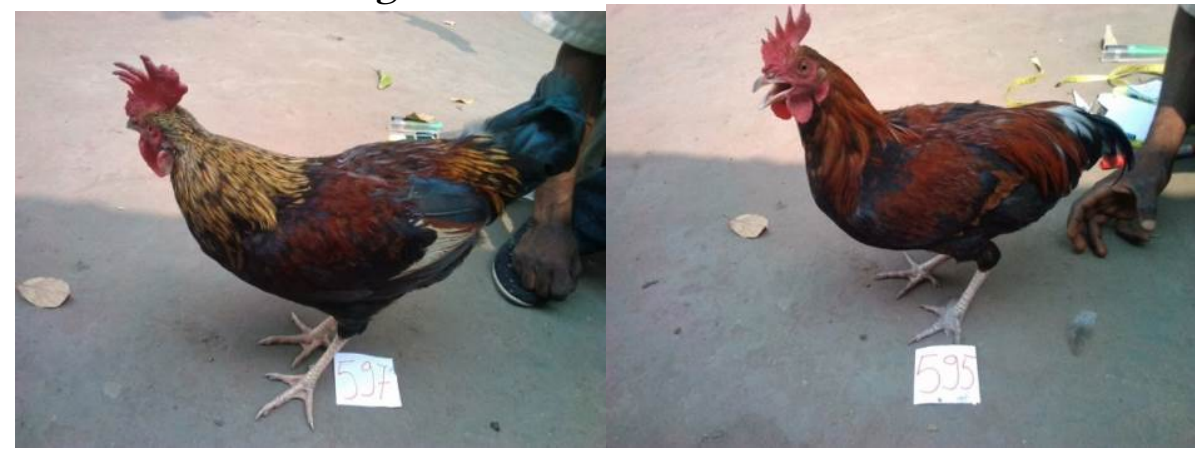

Figure $n^{\circ} 44:$ Photosauracana

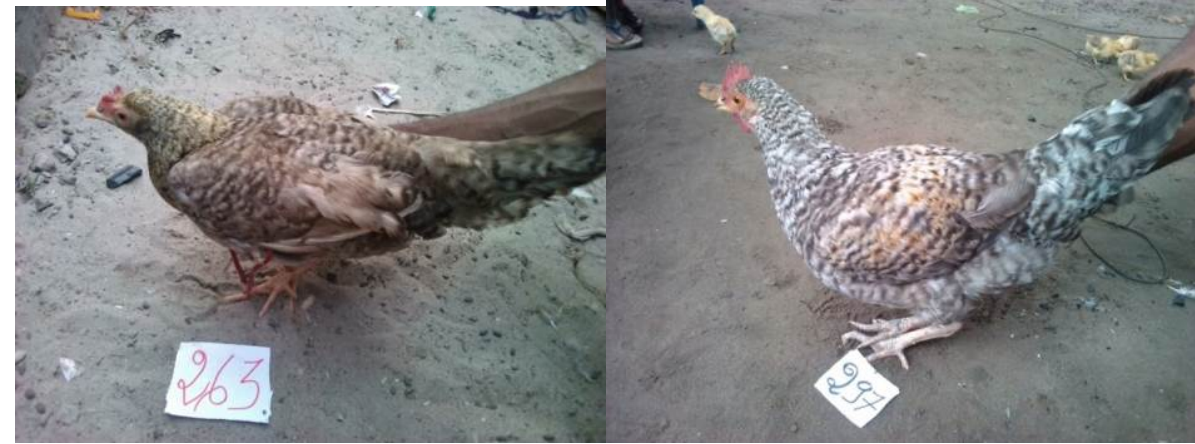

Figure $\boldsymbol{n}^{\circ} 45:$ Photos barré 


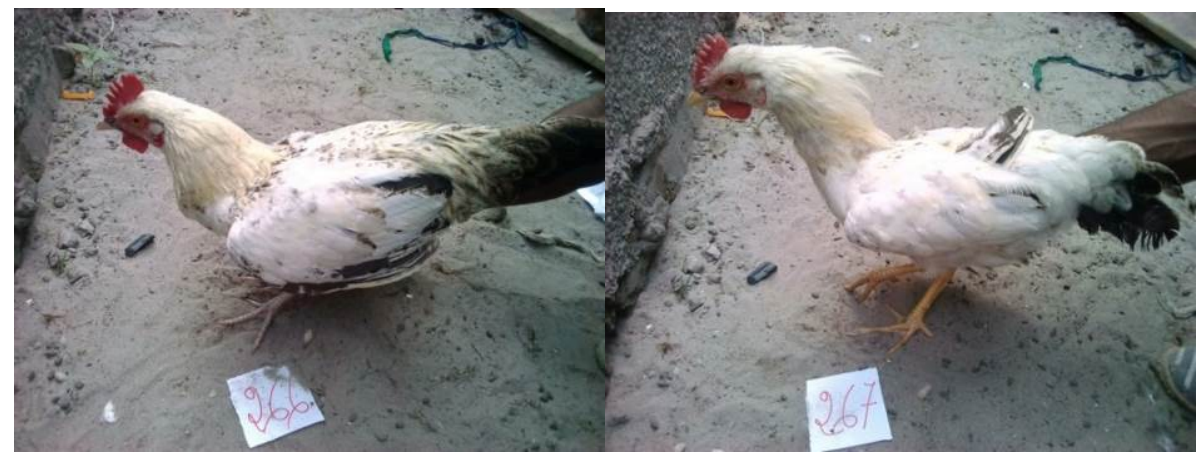

Figure $n^{\circ}$ 46: Photos bleu argenté

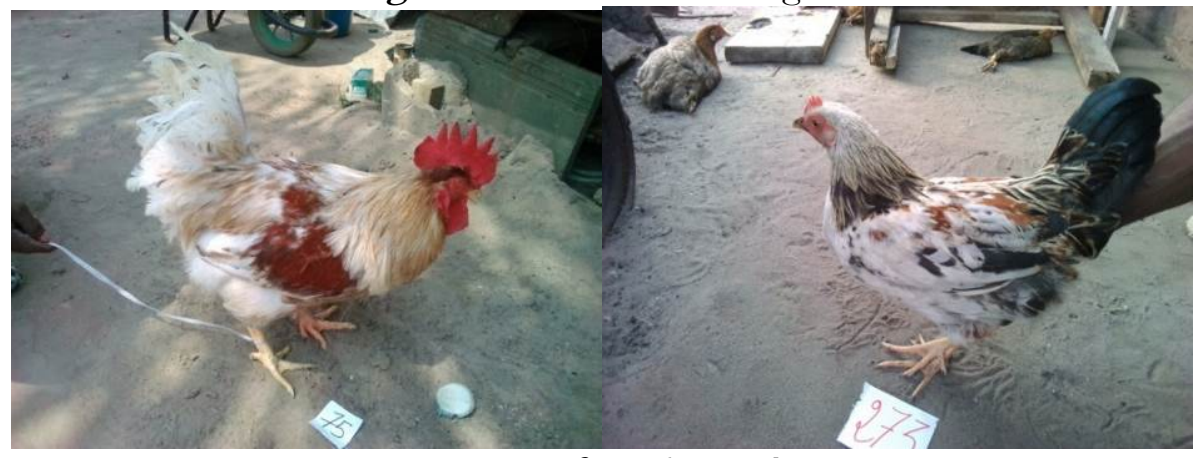

Figure n ${ }^{\circ}$ : Photos pile

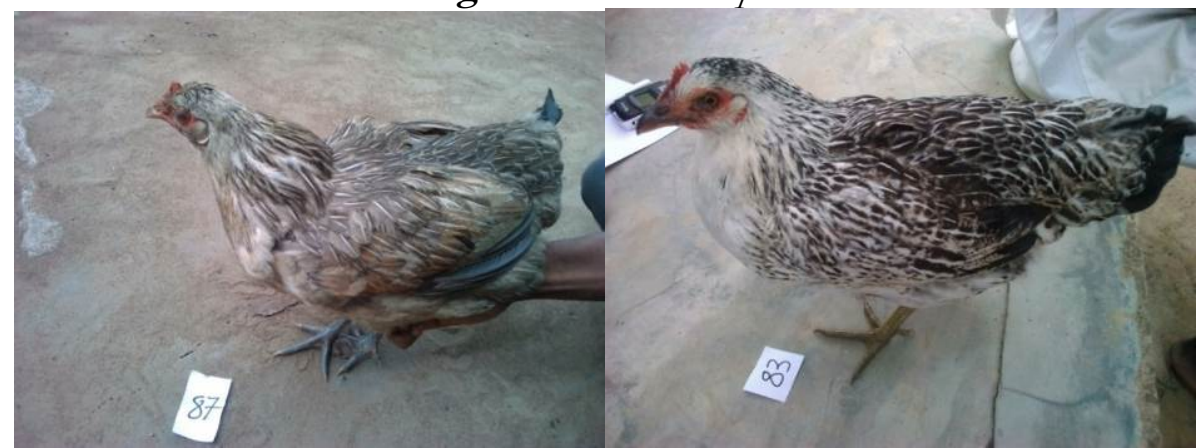

Figure $\mathbf{n}^{\circ} \mathbf{4 8}$ : Photos type perdrix

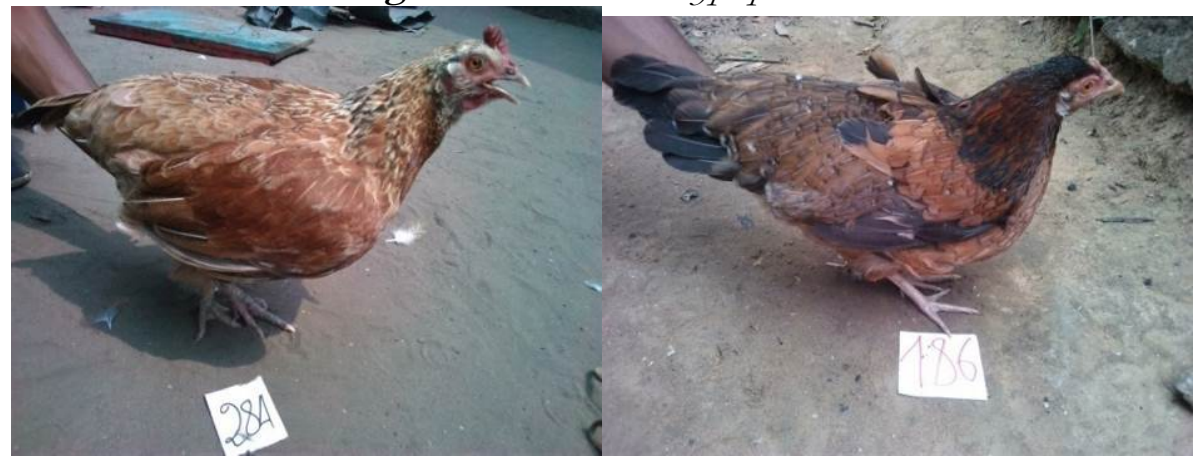

Figure n049: Photos maillé brun 


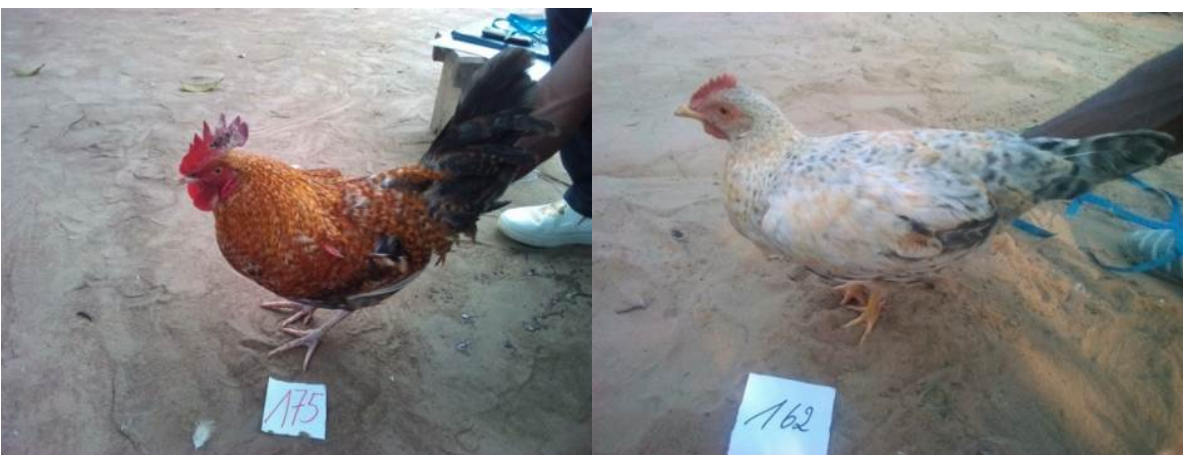

Figure n'50: Photos coucon

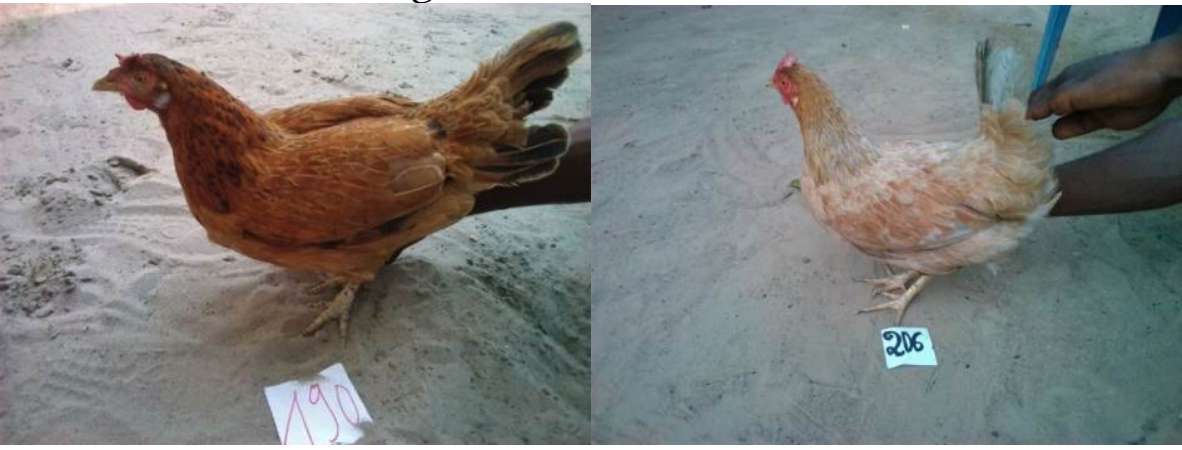

Figure $n^{\circ}$ 51:Photos fauve uni

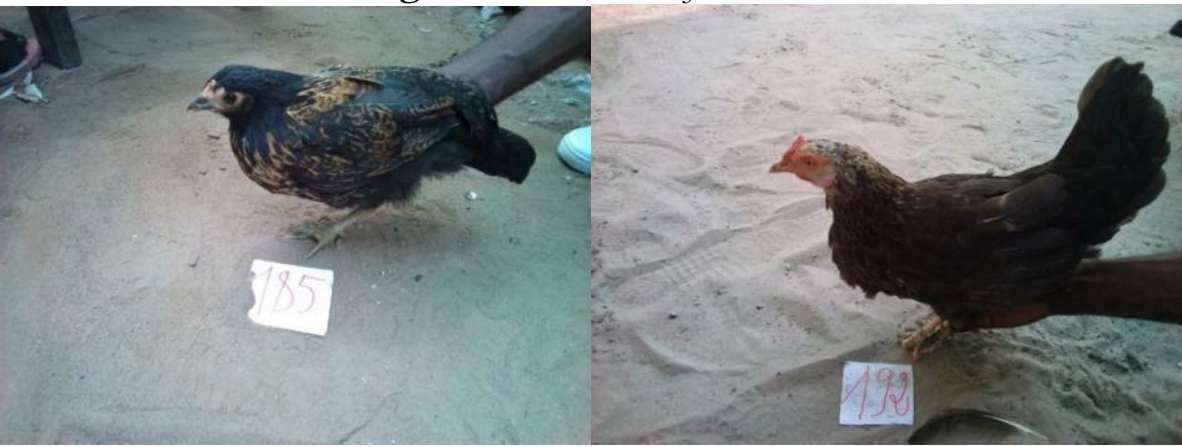

Figure $n^{\circ}$ 52:Photos noir argenté

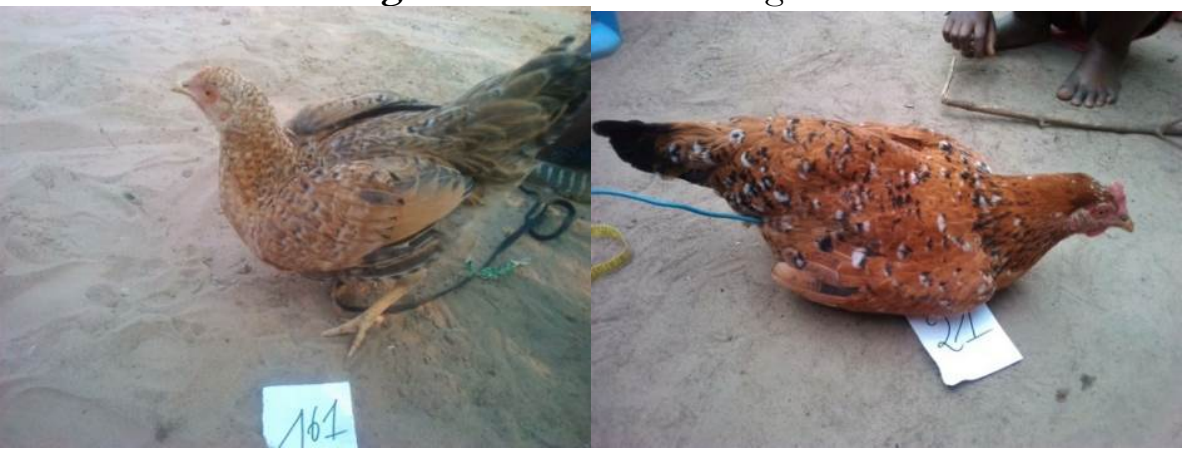

Figure $n^{\circ}$ 53: Photos mille-fleurs 


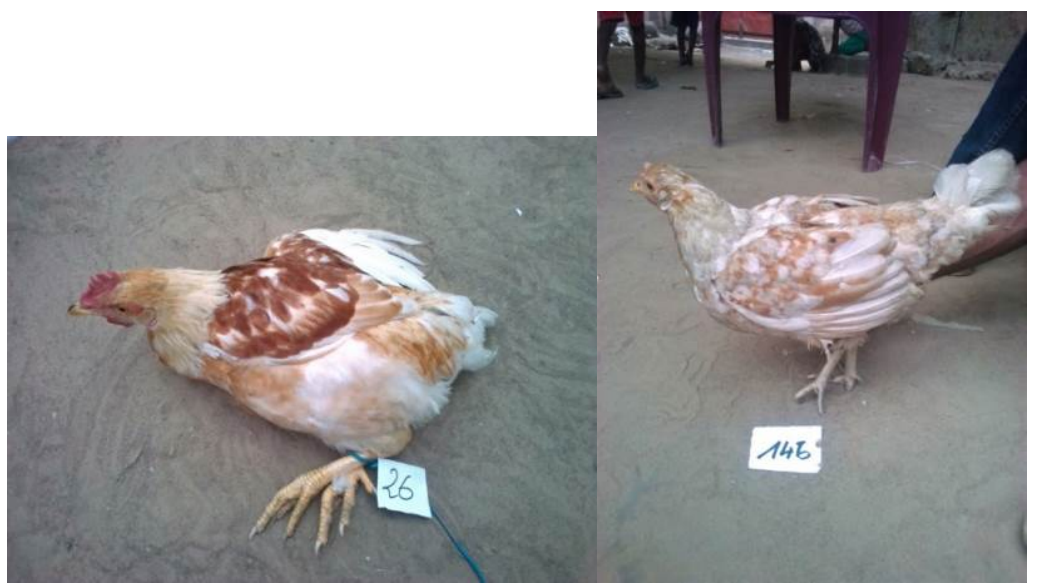

Figure n54: Photos doré à liséré blanc

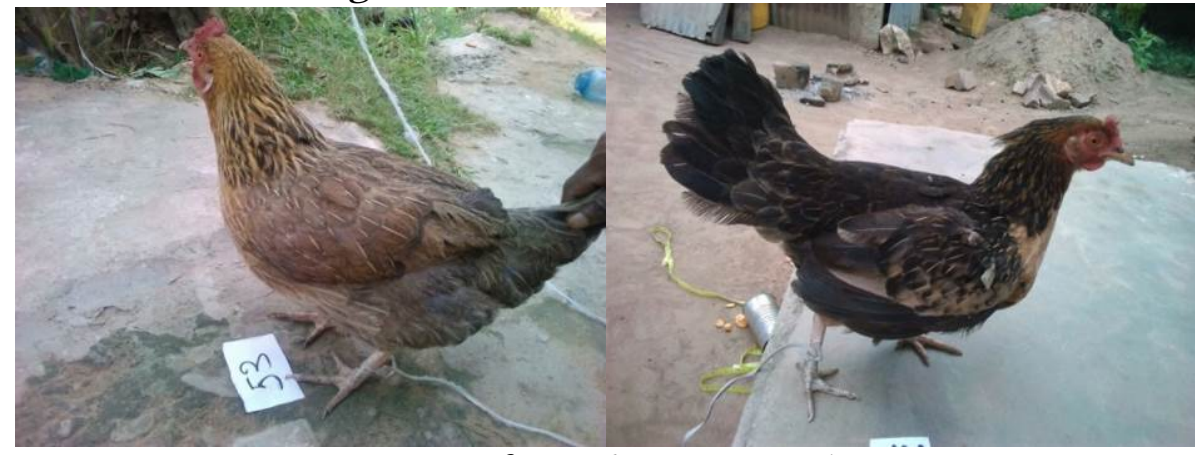

Figure n\$55: Photos saumoné doré

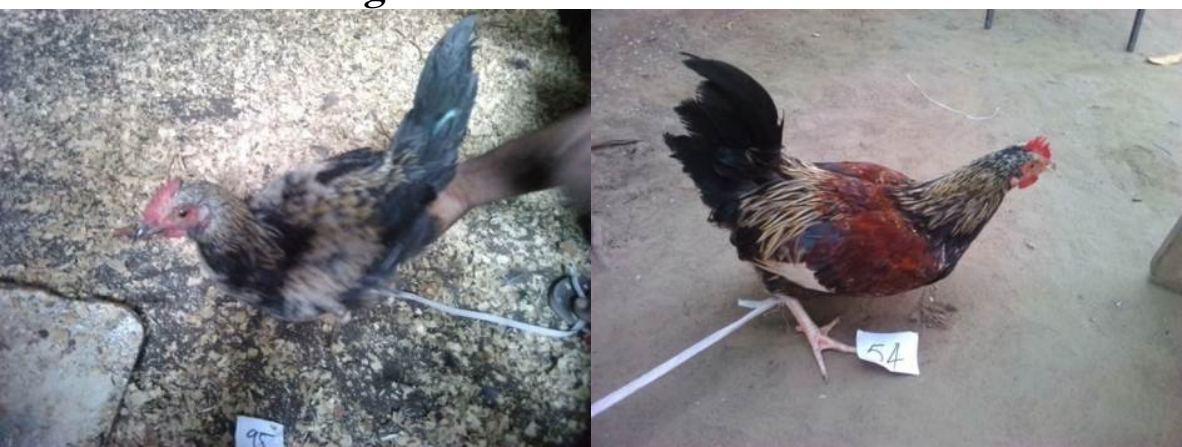

Figure $\mathbf{n}^{\mathbf{5} 56}$ : Photos combattant indien

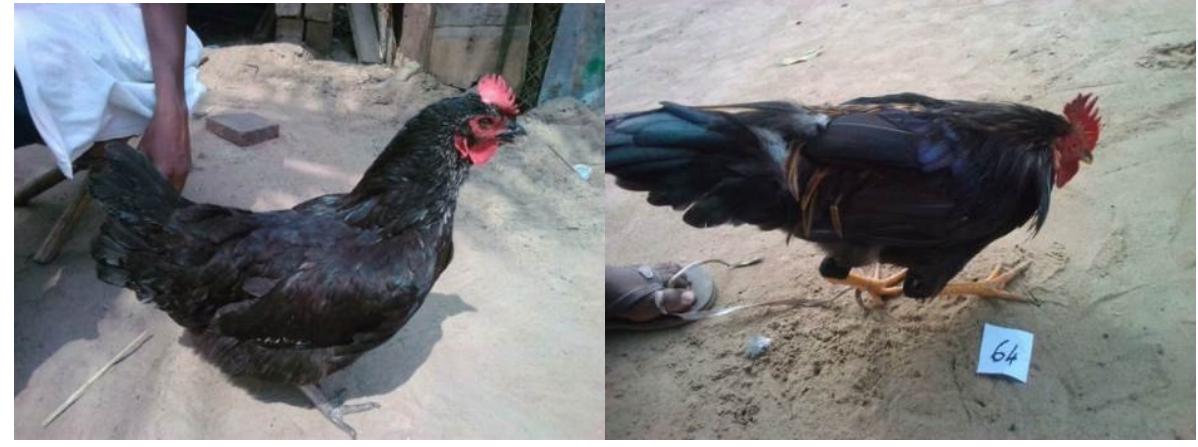

Figure $n^{\circ}$ 57:Photos acajou 


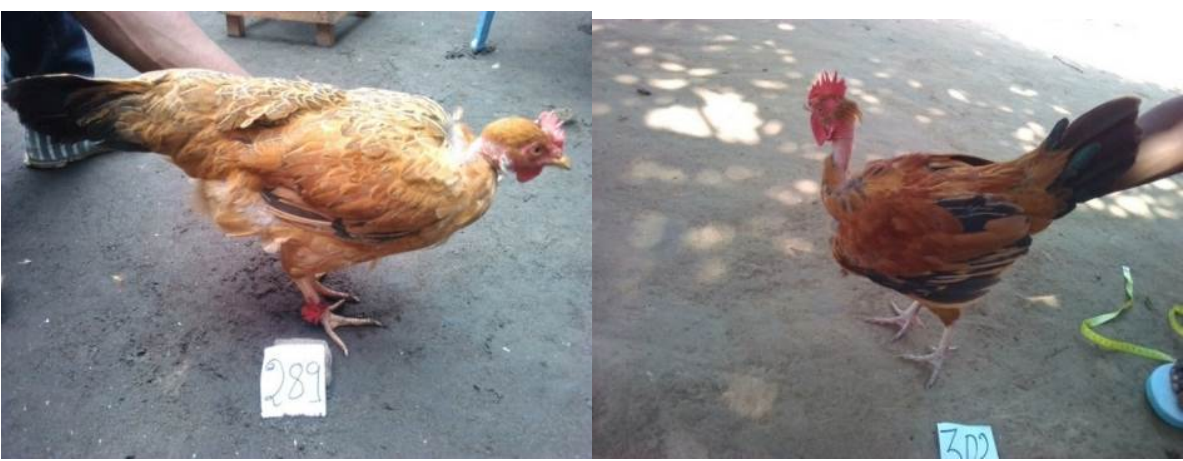

Figure $n^{\circ}$ 58:Photos cou-nu

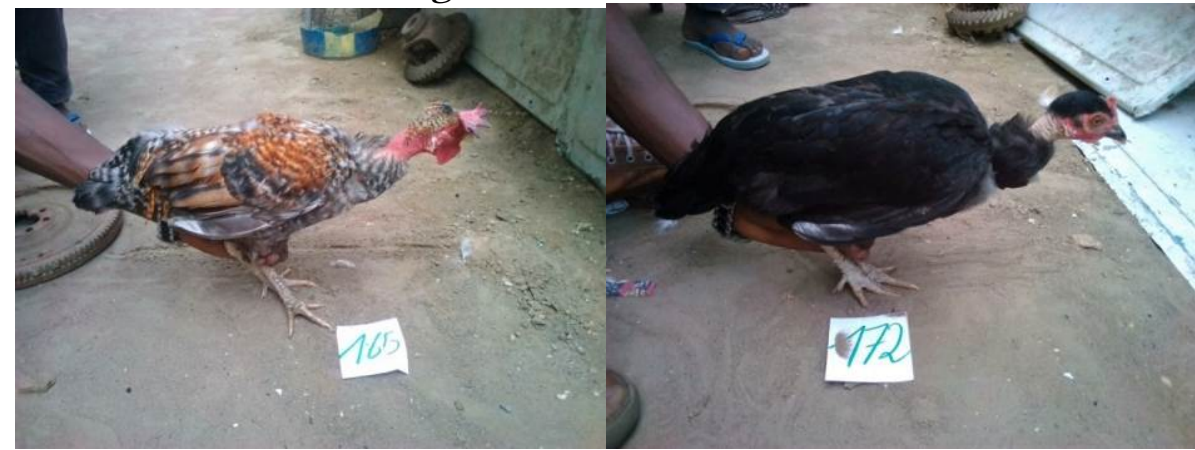

Figure $n^{\circ}$ 59:Photos cou-nu

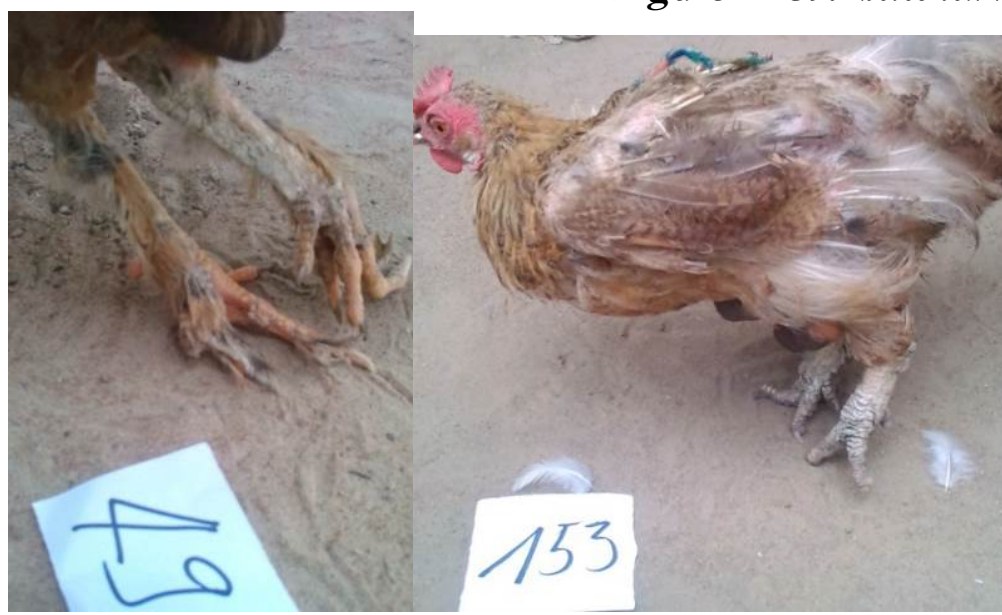

Figure $n^{\circ} 60$ : Photos plumes sur tarse figure $n^{\circ}$ 61: Photos poule avec botte de vautour

L'observation de la distribution de la couleur du plumage chez les poules dans les périphéries nord et sud de Brazzaville a révélé que sur 929 sujets observés, 45 phénotypes ont été dénombrés dans les périphéries nord et sud de Brazzaville. Il s'agit de : blanc 65 sujets $(6,99 \%)$, fauve uni 61 sujets $(6,56 \%)$, blanc tacheté 60 sujets $(6,45 \%)$,blanc herminé 60 sujets $(6,45 \%)$, fauve a queue noir 51 (5,48\%),barré 50 sujets $(5,38 \%)$,coucou 48 sujets $(5,16 \%)$, doré saumoné bleu 45 sujets (4,84\%), type perdrix 36 sujets $(3,87 \%)$, froment 33 sujets (3,55\%),noir étendu 28 sujets (3\%),saumoné doré 28 sujets (3\%), aura cana 28 sujets $(3, \%)$, pile 25 sujets $(2,69 \%)$,wyandotte argenté 25 sujets $(2,69 \%)$, noir à camail doré 24 sujets $(2,58 \%)$, noir argenté 23 sujets $(2,47 \%)$, maillé brun 20 sujets $(2,15 \%)$, blanc sale 21 sujets $(2,26 \%)$, acajou 18 ( $2 \%$ ), kokloyaya 15 sujets $(1,61 \%)$, saumoné foncé 13 sujets $(1,39 \%)$, noir gris perle12 sujets $(1,29 \%)$,mille fleurs 12 sujets $(1,29 \%)$, noir restreint 11 sujets $(1,18 \%)$,noir herminé 9 sujets (1\%),caillouté 9 sujets $(0,96 \%)$,perdrix à maillé doré 9 sujets $(0,96 \%)$, roux à double liséré 9 
sujets ( $0,96 \%)$, doré à liséré noir 8 sujets $(0,86 \%)$, doré à liséré blanc 8 sujets $(0,86 \%)$, doré à liséré blanc 8 sujets $(0,86 \%)$,perdrix maillé argenté 8 sujets $(0,86 \%)$, ardennaise saumoné 7 sujets $(0,75 \%)$, emplument normal 7 sujets $(0,75 \%)$, cou-nu 6 sujets $(0,64 \%)$, leghorn

\section{DISCUSSION}

6.1 Type et coloration de la crête : Les crêtes observées sont de type simple (70\%), double $(23 \%)$; tandis que la couleur rouge de la crête représente $99 \%$ contre $1 \%$ pour la couleur rose pâle. La majorité des études menées à travers les tropiques (Missobou et al., 1998; Juarez et al., 2000 ; Fotsa et al., 2001) indiquent que la crête simple est la plus fréquente. La crête est soit rouge, soit rouge pâle, soit rouge sablé noir, et de types simple ou double (JC Fotsa., 2007). Il a été observé toute fois chez certaines poules, l'absence de crête avec quelques protubérances et la présence de la crête double chez un certain nombre de coqs.

6.2 Couleur et fréquence du bec: L' observation de la couleur des becs fait ressortir qu'ils sont de plusieurs couleurs à savoir le jaune $23,89 \%$, le blanc $22 \%$, le noir $16,57 \%$, le corne $15 \%$, le marron $14 \%$, le crème $7,85 \%$. Les caractéristiques du bec, sont semblables à celles qui sont rapportées pour les poules dans d'autres pays africains (Missobou et al., 1998; Benabdjelil et Bordas ., 2005 ; Keambou et al., 2007).

6.3 Couleur et la forme des barbillons : Nous avons au cours de notre étude observés deux formes de barbillons a savoir, les barbillons de forme ovale qui représentent 99\% contre les barbillons de forme ronde $1 \%$. La couleur des barbillons est unique, (rouge) chez tous les sujets. La dominance de la couleur rouge des barbillons observée chez la souche locale ou Batéké correspond à ce qui a été rapporté par Keambou et al., 2007 au Cameroun ; par Missohou et al., 1998 au Sénégal et Benabdeljelil et Bordas en 2005 au Maroc.

6.4 Couleur des yeux : La couleur des yeux est génétiquement peu étudiée. La couleur rouge orangé des yeux de nos poules, fréquemment observée dans la plus part des études correspond au type sauvage (Mac arthur, argenté 5 sujets $(0,53 \%)$, red cap 5 sujets $(0,53 \%)$, bleu argenté 3 sujets $(0,32 \%)$, silicienne 3 sujets $(0,32 \%)$, combattant indien 3 sujets $(0,32 \%)$, argenté à liséré doré 3 sujets $(0,32 \%)$ ayarakiridjé 2 sujets $(0,21 \%)$, noir cuivré 2 sujets $(0,21 \%)$.

1993). Plusieurs couleurs des yeux ont été observés au cours de notre observation sur le terrain. Les yeux de couleur orange clair représentent $36,67 \%$ contre $21,52 \%$ pour les yeux orange foncé, $16,14 \%$ pour les yeux noir, $13,88 \%$ pour les yeux brun clair et enfin $10,76 \%$ pour les yeux brun foncé. Nous constatons que la couleur orange associée d'autres couleurs domine sur la couleur noir.

6.5 Couleur des tarses : La fréquence des poules à tarses pigmenté noir $(3,98 \%)$ est plus faible que celle des sujets a tarses blanc $(40,6 \%)$, jaune $(36,59 \%)$, gris $(9,25 \%)$, pigmenté jaune $(8,39 \%)$, car il est possible que la présence de la mutation peau blanche et jaune soit mal détectée chez les poules ayant des tarses noires (JC Fotsa., 2010).

Les tarses sont majoritairement blancs. Cependant d'autres couleurs sont aussi représentées tels que le jaune, le gris, le pigmenté jaune et e pigmenté noir.

6.6 Distribution de la couleur du plumage : 45 couleurs de plumage ont été observées chez les populations de poules locales ou Batékés dans les périphéries nord et sud de Brazzaville dont les plus représentatives sont le blanc (65 sujets, soit $6,99 \%$ ) ; fauve uni (62 sujets, soit $6,50 \%$ ) ; blanc tacheté (60 sujets, soit $6,45 \%)$; blanc herminé (60 sujets, soit $6,45 \%$ ) et fauve à queue noir (51 sujets, soit $5 \%$ ). La grande variété des couleurs de plumage est ainsi le résultat de multiples croisements non contrôlés depuis plusieurs décennies entre volailles ayant différents coloris de plumage, qui donnent naissance à d'autres combinaisons existant en faibles proportions (Akonango et al., 2004). Les mêmes couleurs de plumage ont été observées chez la poule Kabyle mais avec des fréquences différentes. La couleur blanche et noire dominent la plus part du temps (Moula et 
al., 2009). Ces variétés de couleur ont aussi été observées dans plusieurs pays africains (Benin, Cameroun, Congo Brazzaville, Sénégal, République démocratique du Congo) avec une répartition différentes (Missohou et al., 1998; Akonango et al., 2004 ; Youssao et al., 2010 ; Fotsa et al., 2010 ; Fatima zohora $M$ et al., 2014). Dans notre étude, le plumage normal est plus fréquent que le plumage cou-nu, tarse emplumé et botte de vautour. La fréquence des autres phénotypes: cou-nu, huppé est faible (Fatima zohora et al., 2014). Par ailleurs, on trouve des phénotypes à tarses emplumés et cou-nu, botte de vautour à des fréquences très faibles. Le

\section{CONCLUSION ET PERSPECTIVES}

Cette étude, réalisée dans les périphéries nord et sud de Brazzaville montre que le matériel génétique animale dans les élevages familiers est caractérisé par un faible potentiel de production et une variation phénotypique énorme (JC Fotsa., 2009). Malgré la forte exposition aux environnements difficiles dans lesquelles elles évoluent, les poules locales ou Batékés jouent un rôle socio-économique très important. La riche diversité génétique semble évoluer d'une manière anarchique. Cette race pourra à son tour être le support d'un plan de sélection animale pour permettre aux éleveurs d'avoir des

\section{REFERENCES BIBLIOGRAPHIQUES}

Akouango F, Mouangou F, Ganongo G., (2004): Phénotype et performances d'élevage chez des populations locales de volailles du genre Gallus gallus au Congo Brazzaville. 6 pages

J.C.Fotsa, Rognon, Tixier-Boichard BM, Coquerelle G, Poné KD, Ngou Ngoupayou, Mandjeli Y et Bordas A., (2010): Caractérisation phénotypique des populations de poules locales (Galus gallus) de la zone forestière dense humide à pluviométrie bimodale du Cameroun. 12 pages

N.Moula, Farnir F, A.Salahi, M.Iguerouada, Leroy $P$ and Antoine-Moussiaux, N.Backyard., (2011a): Aviculture familiale au Bas-Congo, République phénotype cou-nu apparait ici comme le plus répandu chez les individus femelles. La fréquence observée semble plus faible chez nous (moins de 1\%) que dans les autres pays : $1,90 \%$ au Cameroun, (Keambou et al., 2007); $1,94 \%$ au Sénégal (Missobon et al., 1998) et $4,13 \%$ en Algérie (Moula et al., 2009). La présence du type cou-nu chez la volaille constitue un avantage certain en milieux tropicaux. Les présents résultats confirment le signe de la primarité du genre Gallus gallus de l'espèce avicole actuellement disponible et employée par nos paysans (Missohou et al., 1998).

rendements plus importants que ceux obtenue jusqu'ici. La variabilité phénotypique de cette race confère à cette population de poule une place socioculturelle et rituelle important (cérémonie de Nzobi, traitement de certaines maladies). Afin de compléter les informations sur la caractérisation morpho-biométrique de cette population de poules, nous envisageons poursuivre la caractérisation dans les autres arrondissements de Brazzaville (Bacongo, Makelekele, Moungali, Ouenze, Talangaî, Mfilou) et dans le reste du pays.

Démocratique du Congo (RDC). 19 pages.

Guèye., 1998: Village egg and fowl meat production in Africa- Regional Report World's Poultry Science Journal, 54: 7386.

Missohou A,Sow R.S, Ngwe-Asoumouc., 1998: caractéristiques morpho biométriques de la poule du Sénégal. Animal Genetic Ressources Information 24 : 63-69

Fatima Zohra Mahami, Gaouar SBS, TabetAoul NT,Tixier-Boichard M et SaïdiMethar N., (2014): Caractéristiques morpho-biométriques et systèmes d'élevage des poules locales en Algérie occidentale (Oranie). 12 pages 
Coquerelle G., 2000: les poules: diversité génétique visible. INRA, pp. 181.

Juarez CA, Manriquez A Jay, Segura CJC., 2000 :

Rasgos

d'apparenciafenotippicaen la aviculture rural de los municipos de la riberadelLago Patzcuaro, Michoacan, Mexico. Livestock Research for Rural Development, 12 (1).

J.C Fotsa, Pone D.K., 2001 : Etude de quelques caractéristiques morphologiques des poulets locaux du Nord-Ouest Cameroun. Bulletin RIDAF Volume 11 $\mathrm{N}^{\circ}$ 2, juillet-décembre 2001 pp 13-20

Benabdeljelil K, Bordas A., 2005: characterisation of beldichiken and turkeys in rural poultry flocks of Morocco. Current statement and future outlook. Animal genetic Ressource Information, 31:87-95.

Keambou TC, Manjeli Y, Tchoumboue J, Teguia A et Iroume RN., 2007: caractérisation morpho biométrique des ressources génétiques des poules locales des hautes terres de l'ouest Cameroun. Livestock Research for Rural Development 19 : article 107 ;

Mack Arthur., 1993: Sex-linked genes in the fowl. Genetics, 18: 210-22.

Moula N, Antoine-Moussiaux N, Farnir F, Detilleux J et Leroy P., 2009: Réhabilitation socio-économique d'une poule locale en voie d'extinction: la poule Kabyle (Thayazitlekvayel). Annales de Médecine vétérinaire. 153: 178-186 ;

Youssao Iak, Tobada PC, Koutinhouin BG, Dahouda M, Idrisou NP, Bonou GA., 2010: phenotypic characterisation and molecular polymorphism of indigenous poultry populations of the species Gallus of Savannah and Forest ecotypes of Benin. African journal of biotechnology 9 .

J.C.Fotsa., (2009): Etude des systèmes d'élevage et description phénotypique des poules locales (Gallus gallus) en milieu rural 